\title{
Triple-deck analysis of the steady flow over a rotating disk with surface roughness
}

\author{
Claudio Chicchiero \\ Dipartimento di Ingegneria Aerospaziale, Università di Pisa, 56122 Pisa, Italy \\ Antonio Segalini* \\ Linné FLOW Centre, KTH Mechanics, 10044 Stockholm, Sweden \\ Simone Camarri \\ Dipartimento di Ingegneria Aerospaziale, Universitá di Pisa, 56122 Pisa, Italy
}

(Received 14 January 2020; accepted 22 December 2020;

published 19 January 2021)

\begin{abstract}
The effect of surface roughness on the steady laminar flow induced by a rotating disk submerged by fluid otherwise at rest is investigated here theoretically and numerically. A theory is proposed where a triple-deck analysis is applied leading to a fast evaluation of the steady-flow modification due to the rough surface. The theory assumes that the roughness is much smaller than the boundary-layer height and is characterized by a significantly longer length scale (slender roughness). Only the leading-order correction is developed here, corresponding to a velocity-field correction that is linear with the roughness height. The proposed theory neglects some curvature terms (here partially accounted by means of a stretching of the radial coordinate and of a scaling of the dependent variables). Numerical simulations performed with different roughness geometries (axisymmetric roughness, radial grooves, and localized bumps) have been used to validate the theory. Results indicate that the proposed theory leads to a good quantification of the flow modifications due to surface roughness at a very low computational cost. A demonstration of the capabilities of the theory is finally proposed where the statistical effects on the flow due to a random (but statistically known) roughness distributed on the surface of a rotating disk are characterized.
\end{abstract}

DOI: 10.1103/PhysRevFluids.6.014103

\section{INTRODUCTION}

The flow over a rotating disk submerged by an otherwise still fluid is a canonical flow case where an exact solution of the Navier-Stokes equations exists: von Kármán [1] demonstrated that the flow has a self-similar solution and identified the viscous length scale $l_{*}=\left(v^{*} / \Omega^{*}\right)^{1 / 2}$ as the characteristic thickness of the boundary layer over the disk, where $\nu^{*}$ and $\Omega^{*}$ indicate the kinematic viscosity and angular velocity of the disk, respectively. The boundary layer has indeed a constant thickness and the wall-parallel velocity components (the radial, $U$, and the azimuthal, $V$ ) scale linearly with the local radius, $r$, while the small wall-normal component, $W$, is constant along the radial direction and scales with the Reynolds number. A set of ordinary nonlinear differential equations

\footnotetext{
*segalini@mech.kth.se
}

Published by the American Physical Society under the terms of the Creative Commons Attribution 4.0 International license. Further distribution of this work must maintain attribution to the author(s) and the published article's title, journal citation, and DOI. Funded by Bibsam. 
was proposed by von Kármán providing the wall-normal variation of the three-dimensional (3D) velocity profile. The fact that the flow is three-dimensional and allows for an analytical solution has attracted much attention from the research community [2] with several studies about the linear stability of this canonical flow [3-5] that exhibits even an absolute instability [6,7].

While the steady laminar flow is well assessed, the steady flow with surface roughness has been much less analyzed despite its fundamental importance. To the authors' knowledge, two models have been proposed in the last 15 years. The first was developed by Miklavčič and Wang [8] where a slip velocity was introduced in the self-similar von Kármán solution by means of a Robin boundary condition at the surface in the form $U(0)=\lambda d U(0) / d \eta$ (and similarly for the azimuthal component with a coefficient $\zeta$ instead of $\lambda$ ), where $\eta$ is the wall-normal coordinate and $\lambda$ is a coefficient related to the roughness type. The approach is very convenient numerically since the same solver can be used to compute the base flow with and without roughness, with a slightly changed boundary condition. Furthermore, by assuming that the roughness characteristics do not change over the disk surface, a self-similar solution is again possible. The drawback of this model is that it is unclear how to relate $\lambda$ with the surface roughness characteristics and, even more fundamentally, whether such a model is sufficient to provide realistic results. Three years later Yoon et al. [9] proposed a numerical approach to characterize axisymmetric sinusoidal-shaped roughness. The numerical approach is nonlinear and parabolic so it necessitates a dedicated solver that, given the restrictions in the roughness choice, did not spread widely in the research community. Furthermore, the latter method does not provide a self-similar solution and some authors [10] have actually averaged the velocity field to reobtain a radial-independent self-similar base flow.

The detailed knowledge of the steady base flow is of paramount importance to study the effect of surface roughness on the rotating-disk flow, especially in terms of linear stability analysis. By using both models [8,9] several stability analyses have been performed [10-12] but some questions remain open about the accuracy of the estimated base flow: the values of $\lambda$ and $\zeta$ associated to any roughness distribution remain unknown, while the flow modification due to a sinusoidal distribution does not provide information for azimuthally varying roughness distributions, especially when considering the impact of the pressure gradient imposed in the azimuthal direction. Corrections to canonical boundary-layer formulations have been recently proposed by Segalini and Garrett [13] for the rotating sphere and by Segalini and Camarri [14] for the cone. All those corrections were related to the effect of viscosity and outer motions away from the surface. The presence of roughness, however, introduces a new length scale and phenomenology inside the boundary layer that is worth investigating due to the current research interest.

The objective of the present paper is to propose a theory based on a triple-deck theory (TDT) approximation valid at a sufficiently large distance from the disk center. The proposed theory works for any type of roughness that is sufficiently slender and is developed only at leading-order providing an analytic solution equivalent to the one of boundary layers over rough surfaces without Coriolis and curvature effects. Due to its characteristics, the proposed method takes into account the effects of roughness on the flow at a very fast computational speed.

The proposed asymptotic theory is first validated against dedicated direct numerical simulations (DNSs). To this purpose, a set of deterministic roughness distributions has been considered thus identifying a sequence of test cases of increasing complexity. The test cases presented provide a wide range of comparison between TDT and DNS, but are still not representative of a realistic roughness distribution. Indeed, roughness is represented by a complex pattern of ridges and valleys and it is often characterized only in a statistical sense in practical applications. Thus, a comparison between TDT and DNS in a stochastic framework is presented to identify some general features of the flow perturbed by a random roughness distribution.

This paper is structured as follows. Section II reports the notation, the equations, and the relevant scalings of the problem analysed by means of the triple-deck framework in Sec. III. Details of the simulations are reported in Sec. IV, while the comparison for the various analysed geometries is reported in Sec. V. Section VI elaborates on the application of a stochastic approach in order to 
characterize the mean value and standard deviation of the perturbed field for a realistic random roughness distribution. Finally, Sec. VII reports a discussion of the results and some final remarks.

\section{PROBLEM FORMULATION}

Let us consider the flow over a rotating disk with roughness distributed at the disk surface. The distribution of roughness height, $h^{*}\left(r^{*}, \theta\right)=h_{\max }^{*} F\left(r^{*}, \theta\right)$, is characterized by a characteristic maximum height, $h_{\mathrm{max}}^{*}$, and a characteristic length scale, $\Delta^{*}=h_{\mathrm{max}}^{*} /\left|\nabla h^{*}\right|$ where $\left|\nabla h^{*}\right|$ indicates the characteristic slope of the wall surface. Here and in the following dimensional quantities will be denoted with an asterisk. The length $\Delta^{*}$ can be conveniently used as characteristic length scale of the problem: it is expected that $\Delta^{*} \gg \sqrt{v^{*} / \Omega^{*}}=l_{*}$, namely that the roughness length is much longer than the viscous scale of the near-wall region. The dimensionless steady-state equations in the rotating cylindrical reference frame (with radial distance measured from the disk center) are

$$
\begin{gathered}
\nabla \cdot \mathbf{U}=0 \\
\mathbf{U} \cdot \nabla \mathbf{U}+\left(\begin{array}{c}
-V^{2} / r-2 V-r \\
U V / r+2 U \\
0
\end{array}\right)=-\nabla P+\frac{1}{\operatorname{Re}_{\Delta}}\left[\nabla^{2} \mathbf{U}-\frac{1}{r^{2}}\left(\begin{array}{c}
U+2 \partial V / \partial \theta \\
V-2 \partial U / \partial \theta \\
0
\end{array}\right)\right],
\end{gathered}
$$

where the coordinates and velocities are scaled with $\Delta^{*}$ and $\Omega^{*} \Delta^{*}$, respectively, and $\operatorname{Re}_{\Delta}=$ $\Omega^{*} \Delta^{* 2} / \nu^{*} \gg 1$ is the Reynolds number based on $\Delta^{*}$. For the sake of notation, length scales normalized with the viscous length scale, $l_{*}$, will be denoted with the + superscript: for instance $\Delta^{+}=$ $\Delta^{*} / l_{*}=\operatorname{Re}_{\Delta}^{1 / 2}$. Consistently with the adopted normalization, the pressure is scaled with $\rho^{*}\left(\Omega^{*} \Delta^{*}\right)^{2}$. The symbols $U, V$, and $W$ will indicate the velocity components in the radial, azimuthal, and wall-normal directions, respectively. The differential operators in cylindrical coordinates are as usual

$$
\nabla \phi=\left(\frac{\partial \phi}{\partial r}, \frac{1}{r} \frac{\partial \phi}{\partial \theta}, \frac{\partial \phi}{\partial z}\right), \quad \nabla^{2} \phi=\frac{\partial^{2} \phi}{\partial r^{2}}+\frac{1}{r} \frac{\partial \phi}{\partial r}+\frac{1}{r^{2}} \frac{\partial^{2} \phi}{\partial \theta^{2}}+\frac{\partial^{2} \phi}{\partial z^{2}} .
$$

If no roughness is present at the wall, i.e., the wall is smooth, the steady-state self-similar solution of von Kármán [1] is obtained, where the pressure is neglected in the horizontal momentum balances since it is of order $O\left(\mathrm{Re}_{\Delta}^{-1}\right)$. The von Kármán flow is given as

$$
\mathbf{U}_{V K}(r, \eta)=\left[r U_{0}(\eta), r V_{0}(\eta), \frac{1}{\operatorname{Re}_{\Delta}^{1 / 2}} W_{0}(\eta)\right], \quad P_{V K}(\eta)=\frac{1}{\operatorname{Re}_{\Delta}} P_{0}(\eta),
$$

with $\eta=z^{*} / l_{*}=z \Delta^{+}$as the boundary-layer coordinate. The self-similar functions are determined by solving the ordinary differential equations [1]

$$
\begin{gathered}
2 U_{0}+W_{0}^{\prime}=0, \\
U_{0}^{2}-\left(V_{0}+1\right)^{2}+U_{0}^{\prime} W_{0}-U_{0}^{\prime \prime}=0, \\
2 U_{0}\left(V_{0}+1\right)+V_{0}^{\prime} W_{0}-V_{0}^{\prime \prime}=0, \\
P_{0}^{\prime}+W_{0} W_{0}^{\prime}-W_{0}^{\prime \prime}=0,
\end{gathered}
$$

where the primes indicate differentiation in the scaled vertical coordinate, $\eta$. The boundary conditions are $U_{0}=V_{0}=W_{0}=0$ at $\eta=0$ and $U_{0}=V_{0}+1=W_{0}^{\prime}=0$ as $\eta \rightarrow \infty$. The solution to Eqs. (5)-(8) is depicted in Fig. 1 along with a schematic three-dimensional representation of the resulting flow field.

When surface roughness is present, the self-similar von Kármán flow is not expected to be valid anymore. However, it is expected that the steady flow could be reasonably described by the 

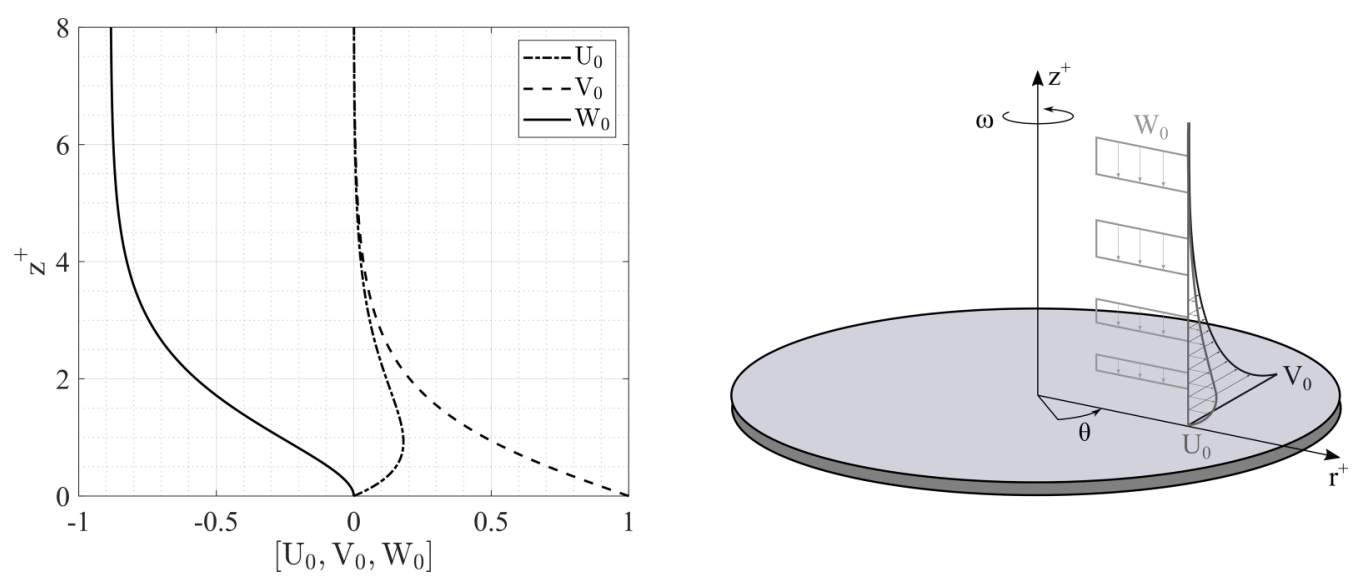

FIG. 1. Self-similar solution of von Kármán flow in the inertial reference frame (left) and three-dimensional representation of the resulting velocity field (right).

asymptotic approximation $\mathbf{U}=\mathbf{U}_{V K}+h_{\max }^{+} \mathbf{u}$, where $\mathbf{u}$ indicates the perturbation velocity due to a unitary maximum roughness height, $h_{\max }^{+}$. The boundary condition of no-slip velocity at the top of the rough surface implies $\mathbf{U}\left[r, \eta=h_{\max }^{+} F(r, \theta)\right]=0$ that can be simplified by translating the boundary condition to the surface as

$$
\mathbf{U}\left(r, \eta=h_{\max }^{+} F\right)=\mathbf{U}(\eta=0)+\left.h_{\max }^{+} F \frac{\partial \mathbf{U}}{\partial \eta}\right|_{\eta=0}+O\left(h_{\max }^{+}\right)^{2}=0 .
$$

By using now the proposed asymptotic approximation, Eq. (9) provides a wall-boundary condition for the perturbation velocity of the form

$$
\mathbf{u}(r, \eta=0, \theta)=-\left.F(r, \theta) \frac{\partial \mathbf{U}_{V K}}{\partial \eta}\right|_{\eta=0}=-F(r, \theta)\left[r U_{0}^{\prime}(0), r V_{0}^{\prime}(0), 0\right] .
$$

It is clear that, in order to ensure the validity of Eq. (10), the roughness height must be $h_{\max }^{+} \ll 1$, therefore ruling out tall roughness elements. It is also worth noting that, since $\Delta$ is defined by the gradient of the roughness height, the derivatives of the scaled roughness height, $F$, with respect to $r$ or $\theta$ are of $O(1)$.

\section{TRIPLE-DECK MODEL}

We consider a bump whose length is equal to $\Delta^{*}$, i.e., longer than the viscous scale $l_{*}$, and we also assume that the bump is located far away from the disk center at a radial position $R^{*} \gg$ $\Delta^{*}$. This suggests the use of the small parameter $\epsilon=\Delta^{*} / R^{*} \ll 1$. These assumptions are not too limiting since stability studies are focused on the transition region [10], $R^{+}$is expected to be quite large anyhow for many analyses of interest, and the roughness is generally much shorter. It is thus convenient to introduce the nondimensional scaled radius $\rho$ as

$$
\rho=\frac{r^{*}-R^{*}}{\Delta^{*}} \longrightarrow r=\frac{1}{\epsilon}+\rho .
$$

Now, since $\Delta^{*}$ is used as reference scale, $R=R^{*} / \Delta^{*}=1 / \epsilon$ is the reference location of the roughness and it is expected to be a large number by hypothesis. The symbol $\rho$ identifies a local radial coordinate that departs from the radial location $r=R$, enabling the local analysis proposed in this section. Furthermore, since $r$ is dominated by the term $1 / \epsilon$, curvature effects are expected 
to be negligible at leading order, so that the roughness element can be considered subjected to a three-dimensional flow rather than to a rotating flow.

By assuming that the bump is localized, it is also convenient to introduce a scaled azimuthal coordinate $\gamma=\theta / \epsilon$ and a vertical stretched coordinate $\zeta=z / \delta$, where the stretching parameter $\delta$ is unspecified at this point to facilitate the deck analysis later on.

The von Kármán base flow (4) can now be expressed in the new coordinates as

$$
\mathbf{U}_{V K}=\left[\frac{1+\epsilon \rho}{\epsilon} U_{0}\left(\Delta^{+} \delta \zeta\right), \frac{1+\epsilon \rho}{\epsilon} V_{0}\left(\Delta^{+} \delta \zeta\right), \frac{1}{\Delta^{+}} W_{0}\left(\Delta^{+} \delta \zeta\right)\right] .
$$

As before, it is assumed that an asymptotic approximation $\mathbf{U}=\mathbf{U}_{V K}+h_{\max }^{+} \mathbf{u}$ applies to the steady flow with roughness together with the boundary condition (10). The continuity equation of the perturbation field can be written as

$$
\frac{\partial u}{\partial \rho}+\frac{\epsilon u}{1+\epsilon \rho}+\frac{1}{1+\epsilon \rho} \frac{\partial v}{\partial \gamma}+\frac{\partial \tilde{w}}{\partial \zeta}=0 \quad \text { with } \quad \tilde{w}=\frac{w}{\delta},
$$

while the linearized momentum equation is

$$
\begin{aligned}
& (1+\epsilon \rho) U_{0} \frac{\partial \mathbf{u}}{\partial \rho}+V_{0} \frac{\partial \mathbf{u}}{\partial \gamma}+\frac{\epsilon W_{0}}{\delta \Delta^{+}} \frac{\partial \mathbf{u}}{\partial \zeta}+\delta \Delta^{+} \tilde{w}\left(\begin{array}{c}
(1+\epsilon \rho) U_{0}^{\prime} \\
(1+\epsilon \rho) V_{0}^{\prime} \\
\epsilon / \Delta^{+} W_{0}^{\prime}
\end{array}\right)+\epsilon\left(\begin{array}{c}
U_{0} u-2\left(V_{0}+1\right) v \\
2\left(V_{0}+1\right) u+U_{0} v \\
0
\end{array}\right) \\
& =-\frac{\epsilon \omega_{p}}{\omega}\left(\begin{array}{c}
\partial p / \partial \rho \\
\left(\begin{array}{c}
\epsilon \rho)^{-1} \partial p / \partial \gamma \\
\delta^{-1} \partial p / \partial \zeta
\end{array}\right)+\frac{\epsilon}{\left(\delta \Delta^{+}\right)^{2}} \frac{\partial^{2} \mathbf{u}}{\partial \zeta^{2}}
\end{array}\right.
\end{aligned}
$$

where $\omega_{p}$ indicates the magnitude of the pressure correction (independent of $\delta$ ) and $\omega$ the magnitude of the velocity correction (function of $\delta$ ). It is worth mentioning that some viscous terms are omitted in the Laplacian since they are negligible at leading order. At this point it is already possible to consider the limit of $\epsilon \rightarrow 0$ obtaining the perturbation equations at leading order. However, by looking at the boundary condition (10), it is clear that the perturbation components in the wallparallel directions should be proportional to the radius $1+\epsilon \rho$. Therefore, the transformations

$$
u=(1+\epsilon \rho) u_{1}, \quad v=(1+\epsilon \rho) v_{1}, \quad \tilde{w}=\tilde{w}_{1}, \quad p=(1+\epsilon \rho) p_{1},
$$

together with the map

$$
\xi=\frac{1}{\epsilon} \ln (1+\epsilon \rho) \longrightarrow \frac{\partial}{\partial \rho}=\frac{1}{1+\epsilon \rho} \frac{\partial}{\partial \xi},
$$

are proposed to account for the radial dependence of the velocity perturbation and to reduce the equations to the form one would have for a boundary-layer flow over bump without curvature terms. Finally, the continuity and momentum conservation equations at leading order can be written as

$$
\begin{gathered}
\frac{\partial u_{1}}{\partial \xi}+\frac{\partial v_{1}}{\partial \gamma}+\frac{\partial \tilde{w}_{1}}{\partial \zeta}=0 \\
U_{0} \frac{\partial \mathbf{u}_{1}}{\partial \xi}+V_{0} \frac{\partial \mathbf{u}_{1}}{\partial \gamma}+\delta \Delta^{+} \tilde{w}_{1}\left(\begin{array}{c}
U_{0}^{\prime} \\
V_{0}^{\prime} \\
0
\end{array}\right)=-\frac{\epsilon \omega_{p}}{\omega}\left(\begin{array}{c}
\partial p_{1} / \partial \xi \\
\partial p_{1} / \partial \gamma \\
\delta^{-1} \partial p_{1} / \partial \zeta
\end{array}\right)+\frac{\epsilon}{\left(\delta \Delta^{+}\right)^{2}} \frac{\partial^{2} \mathbf{u}_{1}}{\partial \zeta^{2}} .
\end{gathered}
$$

Equations (17) and (18) could have been obtained directly from (13) and (14) in the limit of $\epsilon \rightarrow 0$, providing the analogy between an isolated bump over a rotating disk and an isolated bump over a three-dimensional planar boundary layer. Nevertheless, the scalings (15) and (16) lead to the same equations but accounting definitely better for the boundary conditions and partially for the curvature terms as well (even though no Coriolis terms are accounted at the leading order). It is, however, required that $\epsilon \rho \ll 1$ in order to limit the importance of some neglected curvature terms, 


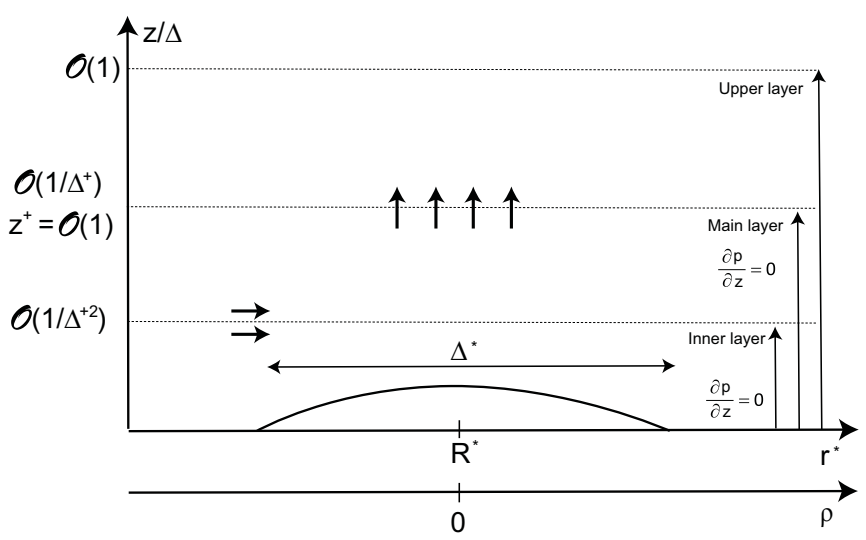

FIG. 2. Schematic description of the triple-deck structure. The thick arrows refer to matching boundary conditions between the various layers.

so that this theory is limited to roughness that does not extend excessively in the radial direction. This is expected to be true for isolated bumps, but it will be shown that the approximation remains good even for roughness distributions extending over several hundreds of viscous units.

One can note that by choosing different $\delta$ values it is possible to magnify different near-wall regions, enabling the development of an analytical solution of the linearized problem by following a triple-deck analysis [15-17]. Here and in the remainder of the section, the triple-deck approach for the three-dimensional boundary layer will be discussed and solved. For the sake of clarity, we provide a schematic description of the proposed triple-deck structure in Fig. 2, which will be addressed to in the following discussion.

It is noteworthy to mention that the triple-deck analysis provides the leading-order solution of Eqs. (17) and (18), and a correction of order $\epsilon^{1 / 3}$ is expected, which does not involve Coriolis or other curvature terms. Nevertheless, this solution is already sufficient to characterize the effect of roughness, as will be discussed in Sec. VIC. Equation (17) is independent of $\delta$ and it is common to all layers, therefore will not be repeated.

\section{A. Upper layer analysis}

The upper layer is characterized by a unitary aspect ratio in the radial and vertical directions, implying that $\delta_{\text {upper }}=1$. Here the pressure gradient balances the advective terms and no viscous effect is present. The von Kármán flow approaches to a constant $\left(0,-1, W_{\infty} / \Delta^{+}\right)$so that several terms of the advective part vanish. The velocity perturbation is of order $\omega_{\text {upper }}$ and balances the pressure-magnitude term, $\epsilon \omega_{p}$. The leading-order equation is indeed

$$
\frac{\partial \mathbf{u}_{1}}{\partial \gamma}=\left(\begin{array}{l}
\partial p_{1} / \partial \xi \\
\partial p_{1} / \partial \gamma \\
\partial p_{1} / \partial \zeta
\end{array}\right),
$$

together with the continuity equation (17). By taking the divergence of the momentum equation (19) one obtains $\nabla^{2} p_{1}=0$ that can be solved by means of the Fourier transform here defined as

$$
\hat{p}_{1}\left(k_{\xi}, k_{\gamma}\right)=\int_{-\infty}^{+\infty} \int_{-\infty}^{+\infty} p_{1}(\xi, \gamma) \exp \left(i k_{\xi} \xi+i k_{\gamma} \gamma\right) d k_{\xi} d k_{\gamma}
$$

so that the solution of the Laplace equations is

$$
\hat{p}_{1}=\hat{\Gamma}\left(k_{\xi}, k_{\gamma}\right) \exp \left(-\sqrt{k_{\xi}^{2}+k_{\gamma}^{2}} \zeta\right)
$$


where $\hat{\Gamma}\left(k_{\xi}, k_{\gamma}\right)$ is a constant determined by the wall condition. The Fourier-transformed wallnormal velocity in this layer is computed as

$$
\hat{w}_{1}=\frac{1}{i k_{\gamma}} \frac{d \hat{p}_{1}}{d \zeta} \longrightarrow \hat{w}_{1}(0)=-\frac{\sqrt{k_{\xi}^{2}+k_{\gamma}^{2}}}{i k_{\gamma}} \hat{\Gamma} .
$$

This vertical blowing velocity cannot be obtained from the boundary condition at the wall [since Eq. (10) prescribes that $w_{1}=0$ ], so that it needs to be determined from what happens within a new layer closer to the wall.

\section{B. Main layer analysis}

While the upper layer is characterized by the far-wall values of the base flow (therefore irrotational), in the main layer the boundary-layer scale is used so that $\delta_{\text {main }}=1 / \Delta^{+}$. By considering that the matching of the vertical velocity at the top of the main layer requires that $\delta_{\text {main }} \omega_{\text {main }}=$ $\delta_{\text {upper }} \omega_{\text {upper }}$, it follows that the magnitude of the velocity perturbation between the two layers is different and that $\omega_{\text {main }}=\Delta^{+} \omega_{\text {upper }}$, implying that pressure gradient effects are of smaller order of magnitude in this layer. Consequently, the leading-order momentum equations in this layer are

$$
U_{0} \frac{\partial u_{1}}{\partial \xi}+V_{0} \frac{\partial u_{1}}{\partial \gamma}+\tilde{w}_{1} U_{0}^{\prime}=0, \quad U_{0} \frac{\partial v_{1}}{\partial \xi}+V_{0} \frac{\partial v_{1}}{\partial \gamma}+\tilde{w}_{1} V_{0}^{\prime}=0,
$$

while the pressure gradient $\partial p_{1} / \partial \zeta=0$ throughout the main layer.

By taking now the wall-parallel divergence of the main layer momentum equations (23), it is possible to get

$$
U_{0} \frac{\partial^{2} \tilde{w}_{1}}{\partial \zeta \partial \xi}+V_{0} \frac{\partial^{2} \tilde{w}_{1}}{\partial \zeta \partial \gamma}=U_{0}^{\prime} \frac{\partial \tilde{w}_{1}}{\partial \xi}+V_{0}^{\prime} \frac{\partial \tilde{w}_{1}}{\partial \gamma},
$$

that, after the Fourier transform, becomes

$$
\left(k_{\xi} U_{0}+k_{\gamma} V_{0}\right) \frac{d \hat{w}}{d \zeta}=\left(k_{\xi} U_{0}^{\prime}+k_{\gamma} V_{0}^{\prime}\right) \hat{w}
$$

with solution given by $\hat{w}=-i\left(k_{\xi} U_{0}+k_{\gamma} V_{0}\right) \hat{A}\left(k_{\xi}, k_{\gamma}\right)$, while the wall-parallel momentum equations provide a direct coupling $\hat{u}=U_{0}^{\prime} \hat{A}$ and $\hat{v}=V_{0}^{\prime} \hat{A}$. The coupling between the constants $\hat{A}\left(k_{\xi}, k_{\gamma}\right)$ and $\hat{\Gamma}\left(k_{\xi}, k_{\gamma}\right)$ can be determined from the matching of the vertical velocity between the main and the upper layer leading to

$$
\hat{A}=\frac{\sqrt{k_{\xi}^{2}+k_{\gamma}^{2}}}{k_{\gamma}^{2}} \hat{\Gamma} .
$$

To determine the value of them, the wall boundary condition (10) needs to be applied. The velocity at the bottom of the main layer approaches $\left[A U_{0}^{\prime}, A V_{0}^{\prime}, 0\right]$, suggesting that $A(\rho, \gamma)=-F(\rho, \gamma)$. However, since $V_{0}^{\prime \prime}(0)=0$, no shear stress would be imposed at the bottom of the main layer in the azimuthal direction due to the roughness presence, which is quite odd. At the wall the boundary conditions are imposed on the velocity components but that region is expected to be dominated by viscous effects, neglected in the rotational inviscid main-layer analysis performed here: this suggests the presence of an inner layer under the main layer where viscous effects should become important.

\section{Inner layer analysis}

The inner region is characterized by the wall-boundary condition and it is of thickness smaller than the viscous scale. That means that the von Kármán velocity distribution can be approximated by a Taylor expansion where the linear terms are the dominant ones. Here it is also required that the 
advective term balances the viscous term, leading to the size of the inner layer as $\delta_{\text {inner }}=\epsilon^{1 / 3} / \Delta^{+}$, while the requirement that pressure gradient is of the same order of magnitude provides the relationship between the various layers

$$
\epsilon \omega_{p}=\omega_{\text {upper }}=\omega_{\text {main }} / \Delta^{+}=\epsilon^{1 / 3} \omega_{\text {inner }} .
$$

Now, since at the top of the inner layer the wall-parallel velocity components match the ones at the bottom of the main layer (see Fig. 2), it follows that $\omega_{\text {main }}=\omega_{\text {inner }}$ leading to

$$
\omega_{\text {inner }}=\omega_{\text {main }}=\epsilon^{-1 / 3} \omega_{\text {upper }}=\epsilon^{2 / 3} \omega_{p} \quad \epsilon=\operatorname{Re}_{\Delta}{ }^{-3 / 2} \longrightarrow \Delta^{*}=\left(l_{*}^{3} R^{*}\right)^{1 / 4} .
$$

It is worth to note that the last relationship is consistent with classical triple-deck theory by introducing a Reynolds number based on $R^{*}$ such as $\operatorname{Re}=\left(\Omega^{*} R^{*}\right) R^{*} / \nu^{*}=\left(R^{*} / l_{*}\right)^{2}$, leading to $\Delta^{*}=l_{*} \operatorname{Re}^{1 / 8}[15]$.

The momentum-conservation equations of the inner layer can be written as

$$
\begin{aligned}
& \alpha \zeta \frac{\partial u_{1}}{\partial \xi}+\beta \zeta \frac{\partial u_{1}}{\partial \gamma}+\alpha \tilde{w}_{1}=-\frac{\partial p_{1}}{\partial \xi}+\frac{\partial^{2} u_{1}}{\partial \zeta^{2}}, \\
& \alpha \zeta \frac{\partial v_{1}}{\partial \xi}+\beta \zeta \frac{\partial v_{1}}{\partial \gamma}+\beta \tilde{w}_{1}=-\frac{\partial p_{1}}{\partial \gamma}+\frac{\partial^{2} v_{1}}{\partial \zeta^{2}}
\end{aligned}
$$

together with $\partial p_{1} / \partial \zeta=0$ throughout the inner layer. The constants $\alpha=U_{0}^{\prime}(0)=0.5102$ and $\beta=V_{0}^{\prime}(0)=-0.6159$ are the derivatives of the von Kármán velocity profile at the wall. The wall boundary condition is obtained from Eq. (10) at leading order so that $\mathbf{u}_{1}(0)=-F(\rho, \gamma)[\alpha, \beta, 0]$ and $\omega_{\text {inner }}=h_{\max }^{+} / \epsilon$.

One can construct an equation for the vertical velocity only by taking the horizontal divergence of the momentum equations and using the continuity equation as

$$
\frac{\partial^{3} \tilde{w}_{1}}{\partial \zeta^{3}}=\alpha \zeta \frac{\partial^{2} \tilde{w}_{1}}{\partial \zeta \partial \xi}+\beta \zeta \frac{\partial^{2} \tilde{w}_{1}}{\partial \zeta \partial \gamma}-\alpha \frac{\partial \tilde{w}_{1}}{\partial \xi}-\beta \frac{\partial \tilde{w}_{1}}{\partial \gamma}-\left(\frac{\partial^{2} p_{1}}{\partial \xi^{2}}+\frac{\partial^{2} p_{1}}{\partial \gamma^{2}}\right)
$$

By taking the Fourier transform of (31), one obtains

$$
\frac{d^{3} \hat{w}_{1}}{d \zeta^{3}}-a \zeta \frac{d \hat{w}_{1}}{d \zeta}+a \hat{w}_{1}=\left(k_{\xi}^{2}+k_{\gamma}^{2}\right) \hat{\Gamma}
$$

with $a=i\left(\alpha k_{\xi}+\beta k_{\gamma}\right)$. By introducing now the coordinate transformation $x=a^{1 / 3} \zeta$, one obtains

$$
\frac{d^{3} \hat{w}_{1}}{d x^{3}}-x \frac{d \hat{w}_{1}}{d x}+\hat{w}_{1}=\frac{k_{\xi}^{2}+k_{\gamma}^{2}}{a} \hat{\Gamma}
$$

Equation (33) can be further differentiated in $x$ leading to

$$
\frac{d^{4} \hat{w}_{1}}{d x^{4}}-x \frac{d^{2} \hat{w}_{1}}{d x^{2}}=0
$$

which is an Airy equation with solution

$$
\frac{d^{2} \hat{w}_{1}}{d x^{2}}=\hat{c}_{1} \operatorname{Ai}(x)
$$

since the second Airy function, $\operatorname{Bi}(x)$, goes to infinity for large values of the argument. The Fourier transform of the vertical velocity is finally obtained as

$$
\hat{w}_{1}=\hat{c}_{1}\left[x M(x)-\mathrm{Ai}^{\prime}(x)+\mathrm{Ai}^{\prime}(0)\right]+\hat{c}_{2} x
$$

with $\hat{c}_{2}=a^{2 / 3} \hat{F}$ according to the continuity equation and the boundary conditions of the wallparallel velocity components, while the function $M(x)$ is given by

$$
M(x)=\int_{0}^{x} \operatorname{Ai}(\xi) d \xi=\frac{1}{3}+\pi\left[\operatorname{Ai}^{\prime}(x) \operatorname{Gi}(x)-\operatorname{Ai}(x) \operatorname{Gi}^{\prime}(x)\right],
$$


where Gi indicates the Scorer function [18]. By substituting now Eqs. (36) into (33) one gets a relationship between the constants $\hat{c}_{1}$ and $\hat{\Gamma}$ as

$$
\hat{c}_{1}=\frac{k_{\xi}^{2}+k_{\gamma}^{2}}{a \mathrm{Ai}^{\prime}(0)} \hat{\Gamma} \text {. }
$$

The radial and azimuthal velocity components can now be obtained by solving the inhomogeneous Airy equations

$$
\frac{d^{2} \hat{u}_{1}}{d x^{2}}-x \hat{u}_{1}=\frac{i k_{\xi}}{a^{2 / 3}} \hat{\Gamma}+\frac{\alpha}{a^{2 / 3}} \hat{w}_{1}(x)=Q_{1}(x), \quad \frac{d^{2} \hat{v}_{1}}{d x^{2}}-x \hat{v}_{1}=\frac{i k_{\gamma}}{a^{2 / 3}} \hat{\Gamma}+\frac{\beta}{a^{2 / 3}} \hat{w}_{1}(x)=Q_{2}(x) .
$$

The solution of equations is found by means of the method of variation of the constants leading to

$$
\hat{u}_{1}=\pi\left[\hat{d}_{1}-\int_{0}^{x} \operatorname{Bi}(\xi) Q_{1}(\xi) d \xi\right] \operatorname{Ai}(x)-\pi \operatorname{Bi}(x) \int_{x}^{\infty} \operatorname{Ai}(\xi) Q_{1}(\xi) d \xi
$$

with

$$
\hat{d}_{1}=\frac{\operatorname{Bi}(0)}{\operatorname{Ai}(0)}\left[\frac{i k_{\xi}}{3 a^{2 / 3}} \hat{\Gamma}+\frac{\alpha}{a^{2 / 3}}\left(\frac{\hat{c}_{1}}{3} \operatorname{Ai}^{\prime}(0)-\hat{c}_{2} \operatorname{Ai}^{\prime}(0)+\hat{c}_{1} \mathrm{Ai}^{2}(0)\right)\right]-\frac{\alpha}{\pi \operatorname{Ai}(0)} \hat{F},
$$

providing the final expression

$$
\begin{aligned}
\hat{u}_{1}= & -\pi \operatorname{Gi}(x) \frac{i k_{\xi} \hat{\Gamma}+\alpha \hat{c}_{1} \operatorname{Ai}^{\prime}(0)}{a^{2 / 3}}-\frac{\alpha \hat{c}_{1}}{a^{2 / 3}}[M(x)+\pi \operatorname{Ai}(0) \operatorname{Bi}(0) \operatorname{Ai}(x)] \\
& -\frac{\alpha \hat{c}_{2}}{a^{2 / 3}}\left[1-\pi \operatorname{Bi}^{\prime}(0) \operatorname{Ai}(x)\right]+\pi \hat{d}_{1} \operatorname{Ai}(x) .
\end{aligned}
$$

Similarly, one could write the expression for the azimuthal velocity as

$$
\hat{v}=\pi\left[\hat{d}_{2}-\int_{0}^{x} \operatorname{Bi}(\xi) Q_{2}(\xi) d \xi\right] \operatorname{Ai}(x)-\pi \operatorname{Bi}(x) \int_{x}^{\infty} \operatorname{Ai}(\xi) Q_{2}(\xi) d \xi,
$$

with constant

$$
\hat{d}_{2}=\frac{\operatorname{Bi}(0)}{\operatorname{Ai}(0)}\left[\frac{i k_{\gamma}}{3 a^{2 / 3}} \hat{\Gamma}+\frac{\beta}{a^{2 / 3}}\left(\frac{\hat{c}_{1}}{3} \operatorname{Ai}^{\prime}(0)-\hat{c}_{2} \mathrm{Ai}^{\prime}(0)+\hat{c}_{1} \mathrm{Ai}^{2}(0)\right)\right]-\frac{\beta}{\pi \operatorname{Ai}(0)} \hat{F} .
$$

In the limit of large $\zeta$, the radial and azimuthal velocity perturbations become

$$
\begin{gathered}
\hat{u}_{\infty}=-\frac{1}{a \zeta}\left[i k_{\xi} \hat{\Gamma}+\alpha \hat{c}_{1} \operatorname{Ai}^{\prime}(0)\right]-\frac{\alpha}{a^{2 / 3}}\left(\frac{\hat{c}_{1}}{3}+\hat{c}_{2}\right), \\
\hat{v}_{\infty}=-\frac{1}{a \zeta}\left[i k_{\gamma} \hat{\Gamma}+\beta \hat{c}_{1} \operatorname{Ai}^{\prime}(0)\right]-\frac{\beta}{a^{2 / 3}}\left(\frac{\hat{c}_{1}}{3}+\hat{c}_{2}\right), \\
\hat{w}_{\infty}=a^{1 / 3} \zeta\left(\frac{\hat{c}_{1}}{3}+\hat{c}_{2}\right)+\hat{c}_{1} \operatorname{Ai}^{\prime}(0)
\end{gathered}
$$

from which it follows that the constant $\hat{A}$ is given by

$$
\hat{A}=-\frac{1}{a^{2 / 3}}\left(\frac{\hat{c}_{1}}{3}+\hat{c}_{2}\right)=-\frac{\hat{c}_{1}}{3 a^{2 / 3}}-\hat{F},
$$

providing the final relationship between the pressure gradient and the bump shape as

$$
\hat{\Gamma}=-\hat{F}\left[\frac{\sqrt{k_{\xi}^{2}+k_{\gamma}^{2}}}{k_{\gamma}^{2}}+\frac{k_{\xi}^{2}+k_{\gamma}^{2}}{3 a^{5 / 3} \mathrm{Ai}^{\prime}(0)}\right]^{-1}
$$




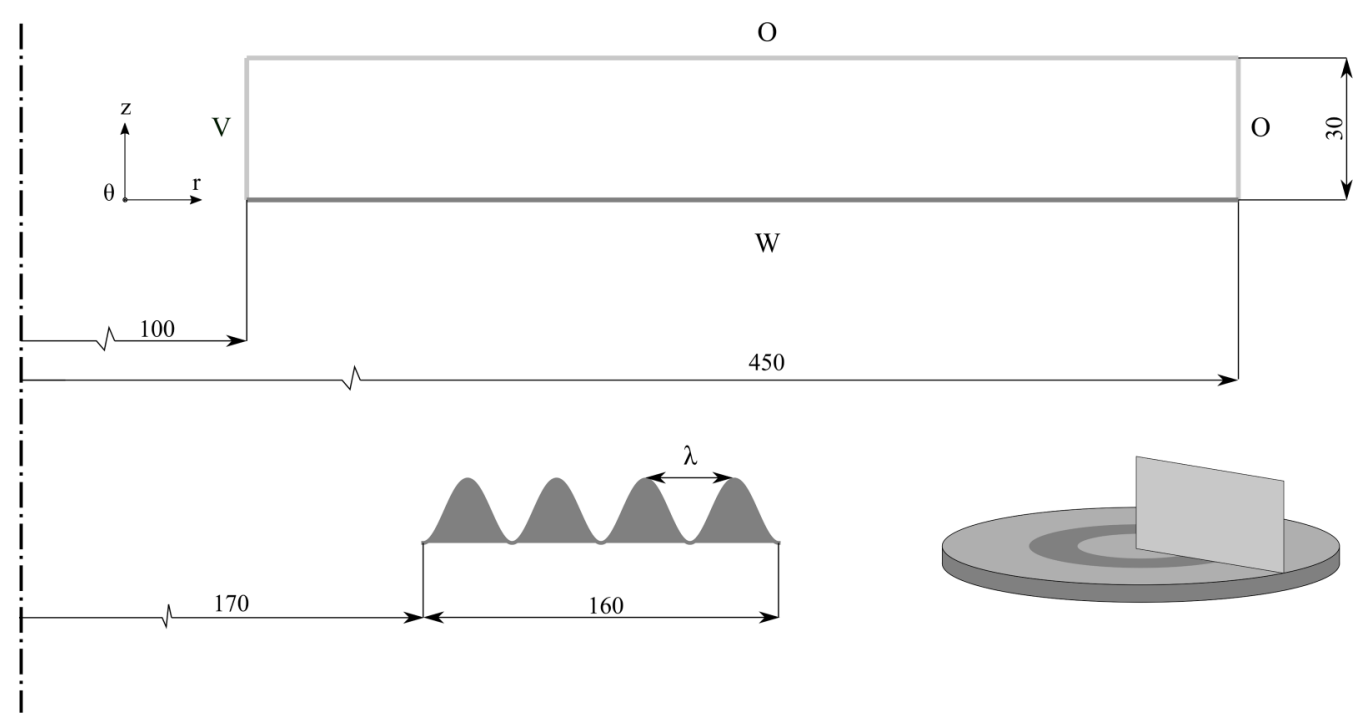

FIG. 3. Computational domain on FREEFEM ++ for the considered axisymmetric groove. The dashed line represents the symmetry axis. Capital letters indicate the applied boundary conditions: (V) von Kármán selfsimilar solution, (O) stress-free condition, (W) no-slip condition. In the lower part of the figure a closeup of the roughness distribution is shown.

Given the bump shape, the determination of the pressure gradient (and of the constants $\hat{A}, \hat{c}_{1}$, and $\hat{c}_{2}$ ) can be performed analytically in the Fourier space. It is worth noting that when $k_{\gamma}=0$ (an axisymmetric bump), no effect can be imposed on the external pressure gradient and $\hat{\Gamma}=0$ from which it follows that $\hat{c}_{1}=0$ and that $A=-F$, as would have followed from an analysis without the inner layer, where the boundary condition should have been imposed directly at the bottom of the main layer.

\section{NUMERICAL SETUP}

In this section the numerical setup employed for evaluating the DNS reference data is presented. Simulations were carried out by considering two different roughness types, namely bumps and grooves, both in axisymmetric and not axisymmetric configurations. Comparison against reference results obtained by DNS also allows for an appraisal of the performance of the method. Tests cases proposed to this purpose are simple enough to highlight the performance and characteristics of the methods, but still representative of the basic elements constituting real rough surfaces. The surface roughness is characterized by an analytical expression in parametric form, specific for each type of studied roughness.

For the axisymmetric configurations, a dedicated Navier-Stokes solver in an inertial reference frame was implemented in FREEFEM++, an open-source finite element solver based on the Galerkin variational formulation [19]. The weak form of axisymmetric Navier-Stokes equations was discretized using classic $P_{2}-P_{1}$ triangular finite elements, to avoid spurious pressure modes. The physical coordinates of the simulation are scaled with the viscous length scale, $l_{*}$, and will just be denoted with a + superscript consistently with the previous section. Furthermore, using the fixed length scale $l_{*}$ rather than $\Delta^{*}$ facilitates the setup and description of the simulation.

The dimensions of the computational domain are fixed for all tested cases. With reference to Fig. 3, the radius $r$ varies between 100 and 450: this range is chosen so as to avoid local instability at $r^{+}=507$ [6]. In the wall-normal direction, the domain height is 30 viscous units, which allows the solution to be practically independent from the domain height, as demonstrated in dedicated 

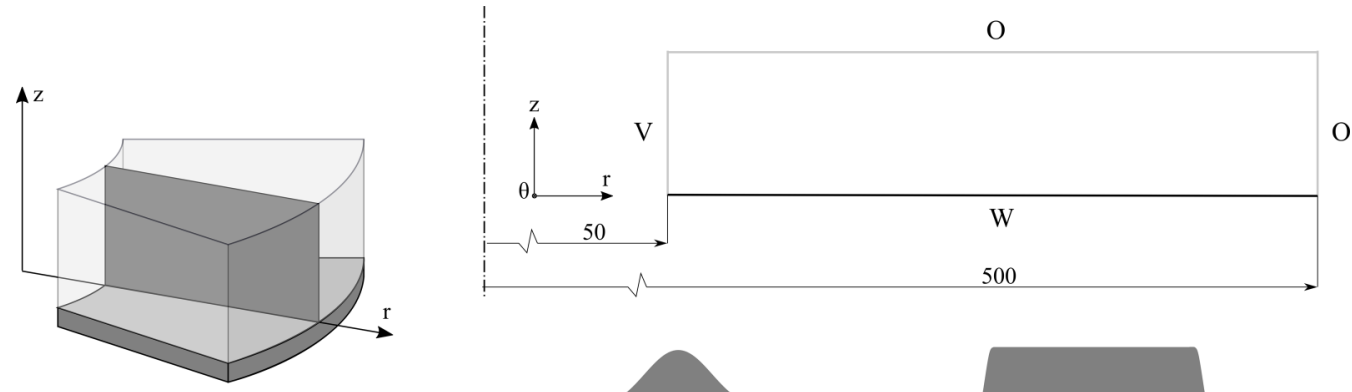

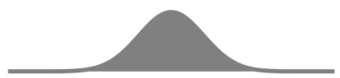

(a)

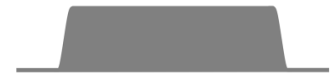

(b)
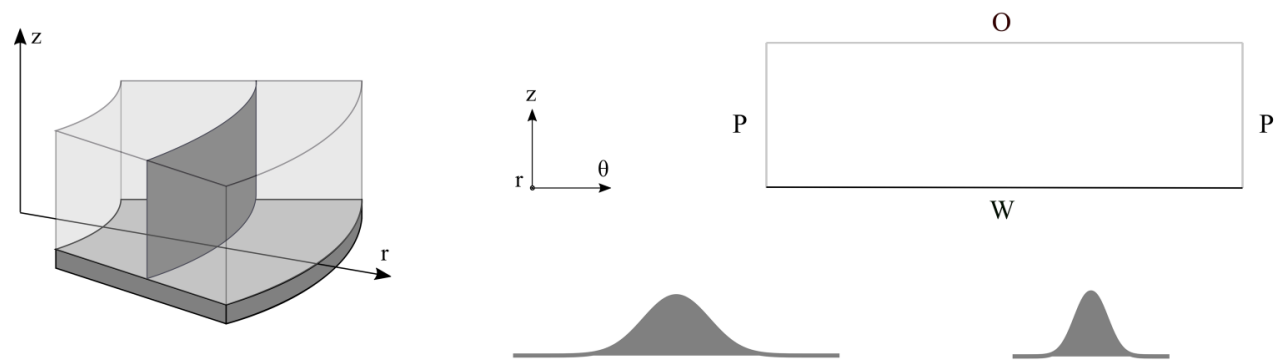

(a)

(b)

FIG. 4. Computational domain projected on the $r-z$ (top) and $\theta-z$ plane. Capital letters indicate the applied boundary conditions: (V) von Kármán velocity solution, (O) stress-free condition, (W) no-slip condition, and (P) periodic (cyclic) condition. The roughness is represented on the $(r, z)$ and $(\theta, z)$ plane for the Gaussian bump (a) and groove $(b)$, respectively.

tests not shown here for the sake of brevity. The von Kármán self-similar solution [1] is imposed at the upstream surface, while the no-slip condition is applied at the wall and stress-free condition on the remaining boundaries, as shown in Fig. 3. The steady solution of the discretized nonlinear system was evaluated by means of a Newton-Raphson method by using the von Kármán self-similar solution as starting point for the iterative solver. In order to have grid-independent results, an adaptive mesh refinement based on intermediate solutions was adopted until the velocity field converged.

As indicated in Fig. 3, the axisymmetric groove starts from $r^{+}=170$ and has a fixed total length in the radial direction equal to 160 ; this particular value has been chosen in order to minimize the effect of outflow boundary conditions on the solution near the grooves. Different wave numbers were considered, providing different length scales, $\Delta^{+}$. The grooves distribution is characterized by

$$
h^{+}\left(r^{+}\right)=\frac{1}{2} h_{\max }^{+}\left[1+\sin \left(\frac{2 \pi r^{+}}{\lambda^{+}}\right)\right]
$$

where $\lambda^{+}$is the groove wavelength, and is varied in the simulations between 2 and 80 , while $h_{\max }^{+}=$ 0.1 for all the cases.

The simulations for the nonaxisymmetric cases were carried out with NEK5000, an open-source spectral element code to solve the Navier-Stokes equations [20]. The solution was evaluated in the rotating reference frame, thus taking into account the centrifugal and Coriolis contributions, by using a $P_{N}-P_{N-2}$ formulation for avoiding spurious pressure modes. The computational domain is an annulus sector and its dimensions are fixed for all cases. With reference to Fig. 4, the annulus radius $r^{+}$varies between 50 and 500, the azimuthal angle $\theta$ varies between 0 and $\pi / 4$ and 
TABLE I. Summary of the mesh final parameters for two nonaxisymmetric geometries in the periodic domain simulations. $N_{r}, N_{\theta}$, and $N_{z}$ indicate number of elements in the radial, azimuthal, and wall-normal direction, respectively, while $N$ is the Legendre polynomial degree.

\begin{tabular}{lrcccc}
\hline \hline Case & $N_{r}$ & $N_{\theta}$ & $N_{z}$ & Elements & $N$ \\
\hline bump & 90 & 40 & 19 & 68400 & 7 \\
groove & 100 & 40 & 19 & 83600 & 7 \\
\hline \hline
\end{tabular}

the wall-normal coordinate, $z^{+}$, ranges between $0-30$. The von Kármán velocity distribution was imposed at the inner radial boundary, no-slip condition at the wall and a von Neumann condition at the outer radial boundary and at $z^{+}=30$. Cyclic boundary conditions were imposed in the azimuthal direction. The grid was locally refined near the bump both in the azimuthal and in the radial directions in order to accurately describe the geometry and the local effect of the bump. The grid convergence of the results was checked using both $\mathrm{h}$ refinement and $\mathrm{p}$ refinement: the final mesh parameters are reported in Table I. The simulation was initialized with the von Kármán self-similar solution and advanced in time with a Courant-Friedrichs-Lewy (CFL) number not larger than 0.3 for iterative stability until the steady state was reached.

The 3D bump is defined, using the previous notation, as a combination of two Gaussian curves, centered at $r_{c}^{+}=250$ and $\theta=\pi / 8$ with a fixed maximum height of $h_{\max }^{+}=0.1$. The shape of the bump is given by

$$
h^{+}\left(r^{+}, \theta\right)=h_{\max }^{+} \exp \left[-\frac{\left(r^{+}-r_{c}^{+}\right)^{2}}{2 \sigma_{r}^{2}}\right] \exp \left[-\frac{(\theta-\pi / 8)^{2}}{2 \sigma_{\theta}^{2}}\right],
$$

where $\sigma_{\theta}$ defines the width in the azimuthal direction. In order to have a circular bump, $\sigma_{\theta}=\sigma_{r} / r_{c}^{+}$. Several values of the bump width have been tested ranging between $2 \leqslant \sigma_{r} \leqslant 8$ to investigate several values of $\Delta^{+}$.

The radial groove extends for 100 viscous units in the radial direction (between $125 \leqslant r^{+} \leqslant 225$ ) and has a constant height equal to $h_{\max }^{+}=0.1$, whereas its shape in the azimuthal direction is defined by a Gaussian curve. Both endings are provided with fillets in order to have a continuous derivative along the radial direction. The final expression is given by

$$
h\left(r^{+}, \theta\right)=h_{\max }^{+} \exp \left[-\frac{(\theta-\pi / 8)^{2}}{2 \sigma_{\theta}^{2}}\right] K\left(\frac{r^{+}-100}{25}\right) K\left(\frac{250-r^{+}}{25}\right),
$$

where $K(x)$ represents the analytical function

$$
K(x)= \begin{cases}0 & x \leqslant 0 \\ {\left[1+\exp \left(\frac{1}{x-1}+\frac{1}{x}\right)\right]^{-1}} & 0<x<1 . \\ 1 & x \geqslant 1\end{cases}
$$

\section{VALIDATION RESULTS FOR AN ASSIGNED GEOMETRY}

The theory proposed in Sec. III can now be validated with the numerical data described in Sec. IV. For the sake of clarity, the reference data are presented by subtracting the von Kármán solution from the total flow field, in order to highlight just the variation of the velocity field with respect to a smooth disk due to the presence of surface roughness. The difference between the simulated field with roughness and the von Kármán flow has been computed at the same height in the absolute coordinate frame relative to the smooth surface. The comparisons between triple-deck theory (TDT) and direct numerical simulations (DNSs) are carried out both for the axisymmetric and nonaxisymmetric cases, in order to show strengths and weaknesses of the proposed theory. 


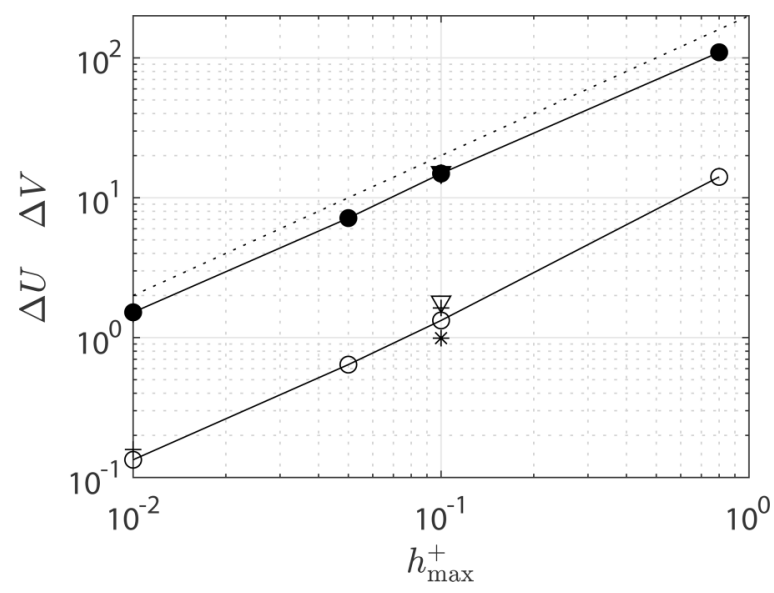

FIG. 5. Maximum azimuthal (filled symbols) and radial (empty symbols) velocity variation due to a bump with different values $h_{\max }^{+}$and $\sigma_{r}$ from the available numerical simulations. $(*) \sigma_{r}=0.5,(\circ) \sigma_{r}=1,(+) \sigma_{r}=2$, and $(\nabla) \sigma_{r}=4$. The dotted line indicates a general linear relationship.

Even though the triple-deck decomposition is developed specifically for localized disturbances, in the following it is also applied for extended ones (such as the axisymmetric or the radial grooves), in order to show the accuracy provided in such cases. Since the cost of the axisymmetric reference simulations is quite low, a preliminary test was carried out to assess the range of roughness heights where the linearized theory is still able to provide reasonable estimations. The details of the tested geometry are reported in Appendix A. A comparison between the maximum absolute variation (with respect to the von Kármán flow) for the radial and azimuthal velocity components with respect to different bump heights is shown in Fig. 5. It is interesting to see that, for relatively small heights, a linear relationship is valid between $h_{\max }^{+}$and the induced velocity, thus confirming that a model that is linear with respect to the height is an excellent approximation of the true behavior. By increasing $h_{\max }^{+}$, the velocity perturbation follows reasonably well the linear behavior, even for the extreme case of $h_{\max }^{+}=0.8$, namely with a bump as thick as the boundary layer, showing that the theory would provide reliable results even beyond the validity limits assumed for its derivation or the case at issue. This is confirmed in Appendix A, where additional comparisons between the predictions of the TDT against reference DNS are reported. However, since it is not expected that the theory should work in general for too large roughness heights, the focus of the following discussion will be kept on roughness elements with small $h_{\max }^{+}$, a case that is more relevant for surface roughness.

The results for the axisymmetric sinusoidal groove are shown in Fig. 6 for two different wavelengths, i.e., $\lambda=4$ and $\lambda=10$, respectively, and $h_{\max }^{+}=0.1$. The comparison indicates that the TDT approach provides a reasonable approximation of the simulation results as for the case of the axisymmetric bump in Appendix A. It is worth to mention that without the variable normalisation proposed in Eqs. (15) and (16), the streamwise evolution of the perturbation velocity in the tripledeck result would be absent, providing a much poorer agreement away from the reference point, $r_{c}^{+}=R^{+}=250$. Even if not important from an applicative viewpoint, it is also interesting to note that the numerical solution shows a merging between the different wave crests at large distance from the surface, while this goes against the linear approximation of the theory, that shows distinct waves throughout the wall-normal direction.

A final remark can be highlighted from this idealized case with sinusoidal roughness. The TDT predicts the velocity distribution over the roughness element but the correction vanishes when the velocity field is averaged over a period since the correction is proportional to the roughness height in the two-dimensional case, as discussed in Sec. III. The numerical simulations instead account for higher-order effects and do not average to zero but to a slight modification of the von Kármán 

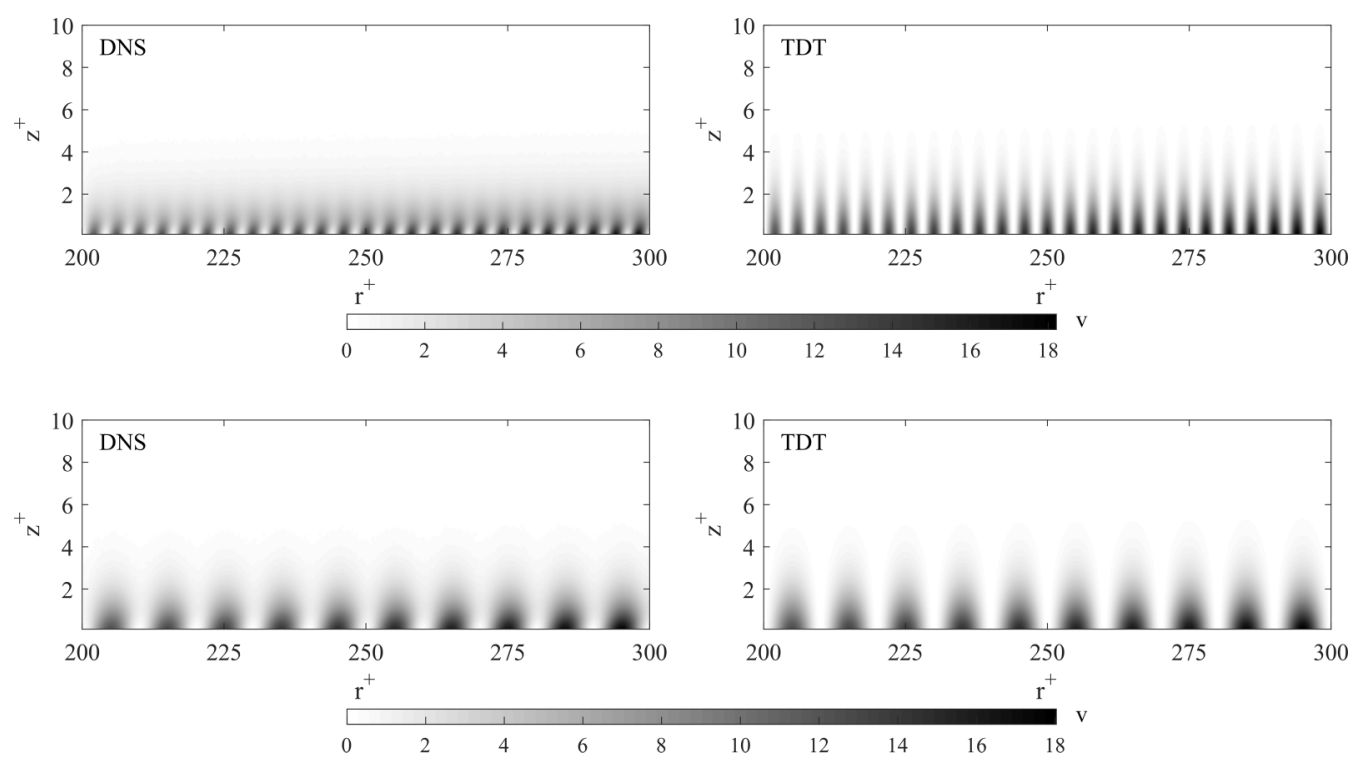

FIG. 6. Azimuthal velocity variation due to an axisymmetric groove with $h_{\max }^{+}=0.1$ and $\lambda=4$ (top) or $\lambda=10$ (bottom) from the DNS and the TDT.

flow similar to what proposed by Miklavčič and Wang [8]. Interestingly, the average becomes independent of the wavelength for $\lambda>4$ and the resulting steady flow can be associated to a Robin condition of the form $U_{0}(0) \approx-0.01 d U_{0}(0) / d \eta$ and $V_{0}(0)=0$. No further tests were performed here to investigate/assess the constants used in the Miklavčič and Wang [8] model for other values of $h_{\max }^{+}$or other roughness configurations.

The two-dimensional cases were characterized by the absence of an azimuthal pressure gradient due to the displacement imparted by the roughness elements. However, the TDT can account for a pressure gradient but only if a three-dimensional roughness element is present. Therefore, it is worthwhile to analyze the theory in the three-dimensional case to assess the importance of pressure gradients effects.

Figure 7 shows the radial and azimuthal components for the center plane of a 3D isolated bump with $\sigma_{r}=4$ and $h_{\max }^{+}=0.1$. In this case, the TDT results are generally in good agreement with the numerical simulations, even if the weak speed-up above the roughness element (for instance at $z^{+} \approx 2$ ) is not properly predicted. This trend is evident by comparing the variation of velocity profiles along the azimuthal direction at a fixed value of $r^{+}$. In Fig. 8 it can be seen that the azimuthal component evaluated with TDT agrees with the one obtained from DNS, while the radial one is slightly below the DNS results, particularly on top of the bump. This could be accounted for in the TDT with a higher-order correction of the main region in light of the weak slip provided by the upper layer on the main one, but this has not been investigated in the present work. It is also noteworthy to point out that, even for a roughness element with $h_{\max }^{+}=0.1$, the wall-normal component of the perturbation spans up to $z^{+}=20$, while the radial and azimuthal components tend to zero for $z^{+}>6$. The discrepancy between DNS and TDT for this component is nevertheless small when compared to the magnitude of the wall-parallel components.

By looking at the $r-\theta$ plane shown in Fig. 9 at $z^{+}=0.2$, it can be seen that the comparison of the theory against the numerical results reveals a good agreement with localized disturbances, and the reader is reminded that in this case the TDT accounts also for the pressure gradient, as highlighted by the shape of the perturbation field.

The resulting pressure correction is depicted in Fig. 10 for the horizontal plane at $z^{+}=0.2$. Even though the proposed theory is developed only at the leading order, the pressure correction evaluated 

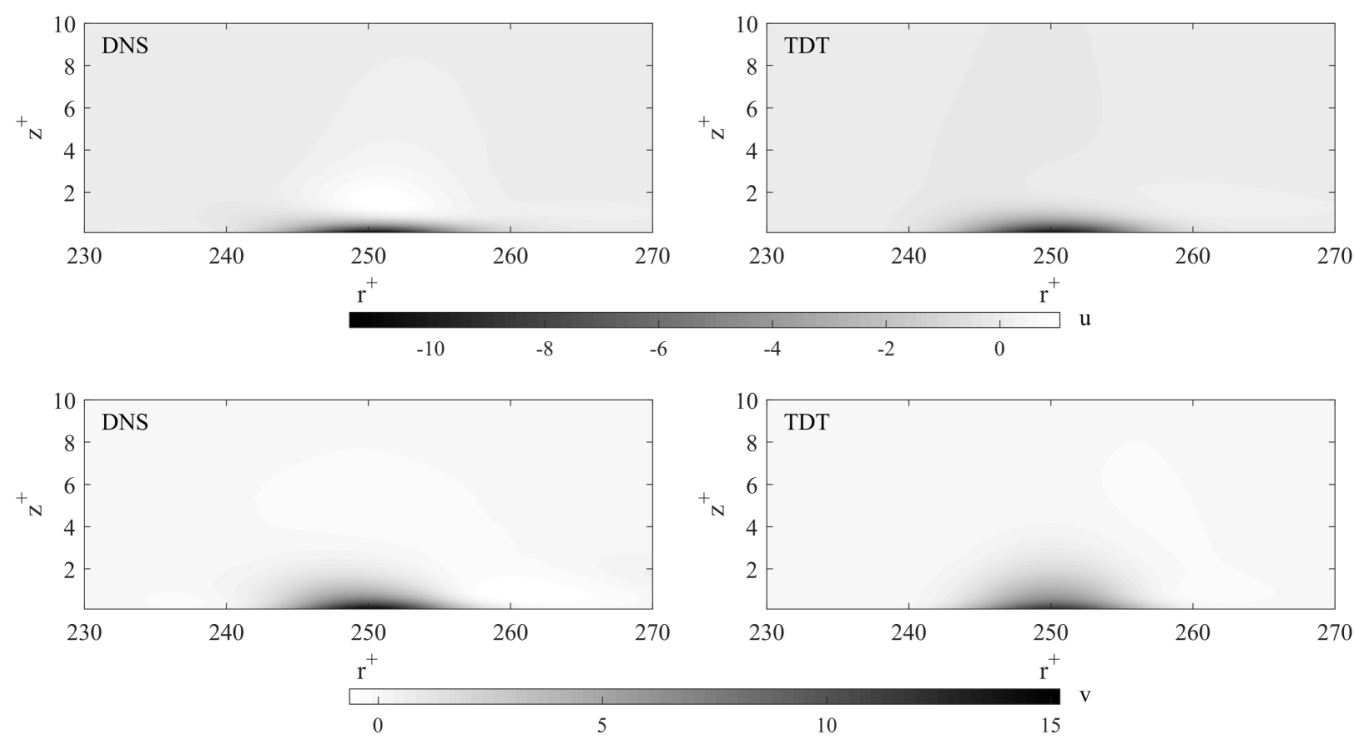

FIG. 7. Radial (top) and azimuthal (bottom) components of the perturbation for the vertical plane along the isolated bump with $\sigma_{r}=4$ from the DNS and the TDT.

with the TDT shows the same trend as for the DNS, while a slight difference arises with respect to the magnitude.

The velocity variation for the radial groove is shown in Figs. 11 and 12 for the center and horizontal planes $\left(z^{+}=0.2\right)$, respectively. Similarly to the axisymmetric sinusoidal groove, the proposed theory is able to predict the overall trend of the velocity variation, but looses reliability when moving away from the reference radial location. Despite the fact that the solution appears less accurate for $z^{+}=1$, it has to be considered that the correction becomes less significant if compared to the boundary-layer velocity while moving along the wall-normal direction, so that it is progressively less important as $z^{+}$is increased. Moreover, by focusing on Figs. 11 and 12, it is possible to conclude that, for roughness elements elongated in the radial direction as grooves, the TDT model shows an evolution due to the local normalization, as explained in Sec. III.

As final remark, it has to be considered that the proposed theory allows for a significant decrease of the computational time while the results are still reliable. For instance, the reference data for the three-dimensional axisymmetric bump required almost 3000 hours of cpu time, while the theoretical calculations ran within a minute. Moreover, the TDT does not depend on the complexity of geometry, while for the DNS the necessity of local refinement for complex surface roughness implies a rapid increment of the computational cost and numerical complexity, especially in the case of structured grids.

\section{STOCHASTIC ANALYSIS FOR RANDOM ROUGHNESS}

The results of previous section showed an overall view of the TDT capabilities and highlighted the fast evaluation of the perturbation field with a good agreement with the reference DNSs. However, surface roughness is usually formed by a complex pattern of ridges and valleys that varies according to different causes, as for instance the manufacturing process. From a practical point of view, it is usually unnecessary or even impossible to characterize the roughness in a deterministic way, thus a statistical description is generally adopted. Many different parameters can be used to describe the surface finish of a workpiece, but one of the most used among them is the arithmetic mean deviation from the mean line $\left(R_{a}\right)$, which can be combined with the root mean 

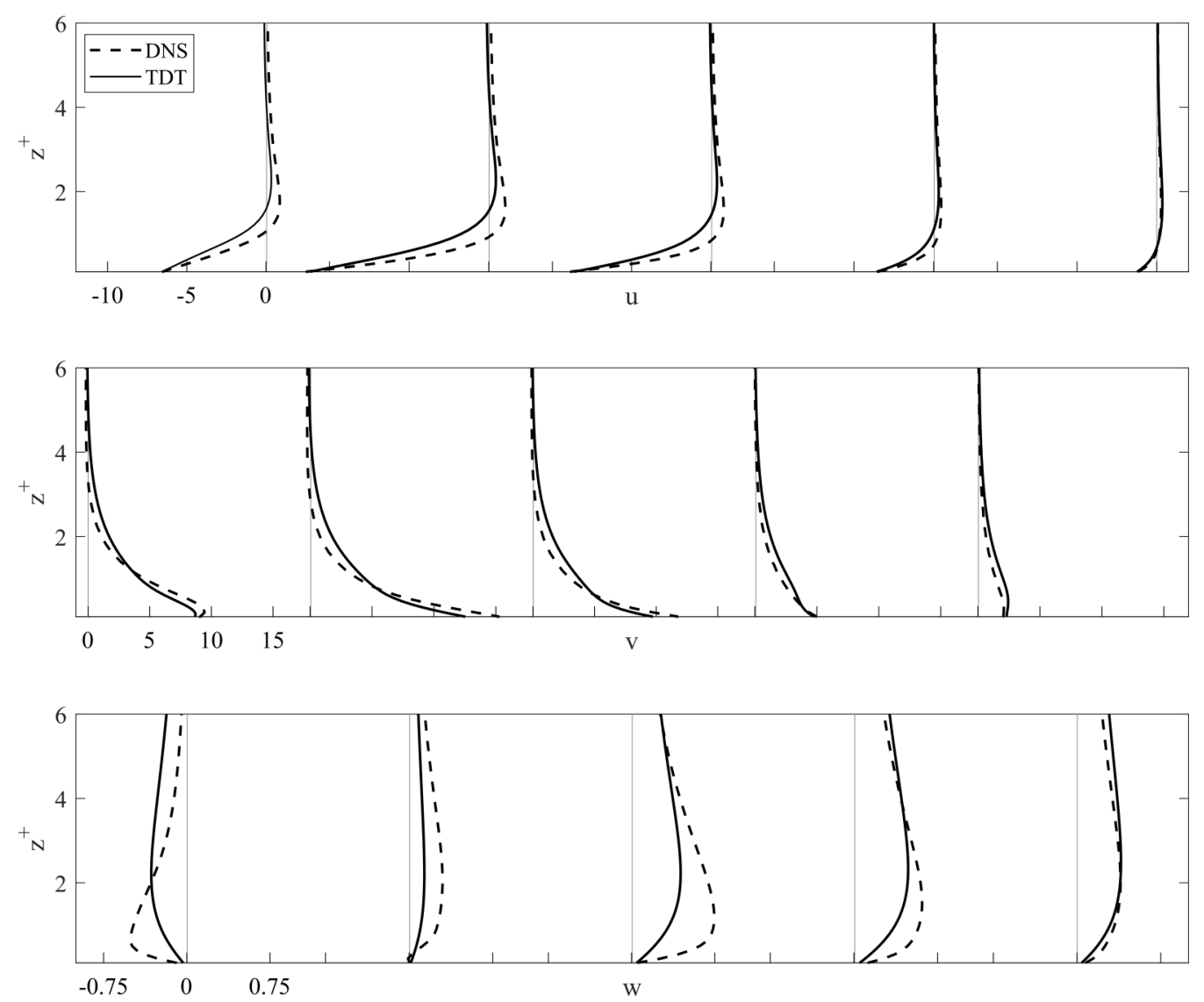

FIG. 8. Comparison of velocity perturbation evaluated by TDT and DNS: radial (top), azimuthal (middle), and wall-normal (bottom) component at five different positions $\left(r^{+}=250, \theta\right)$. From left to right $\theta=$ $[44.1,45,45.7,46.4,46.9]^{\circ}$. The axis values are fixed for each component, thus they are reported only for the first column. The gray vertical line highlights the zero value on the $x$ axis for each position.

square $\left(R_{q}\right)$ of the deviation. Due to the aleatory nature of roughness in real world applications, it seems natural to follow a stochastic procedure in order to consider the height distribution, $h(\mathbf{x})$, not as a given function but as a random variable with assigned mean value and standard deviation. In the following, the analytical relation between the statistics of the perturbation $\mathbf{u}_{1}(\mathbf{x})$ and those of the height distribution, $h(\mathbf{x})$, for the TDT is derived. The setup for the polynomial chaos in order to test the resulting relation against the statistics obtained for the DNS will be further discussed. Hereafter, $\mu_{Q}$ and $\sigma_{Q}$ will refer to the mean value and the standard deviation of a generic quantity $Q$, while $p(Q)$ indicates the probability density function of $Q$.

\section{A. Implications of the TDT}

As reported in Sec. III, in the Fourier space $\hat{\mathbf{u}}_{\mathbf{1}}=\hat{K}_{e}(\mathbf{k} ; z) \hat{F}(\mathbf{k})$, where $\hat{F}$ is the Fourier transform of the normalized $h(\mathbf{x})$ and $\mathbf{k}=\left[k_{\xi}, k_{\gamma}\right]$ is the wave-number vector. According to the convolution theorem, the former relation in the Fourier space can be rewritten in the physical space as a convolution integral and, since one is interested in the statistics of the perturbed velocity, the 

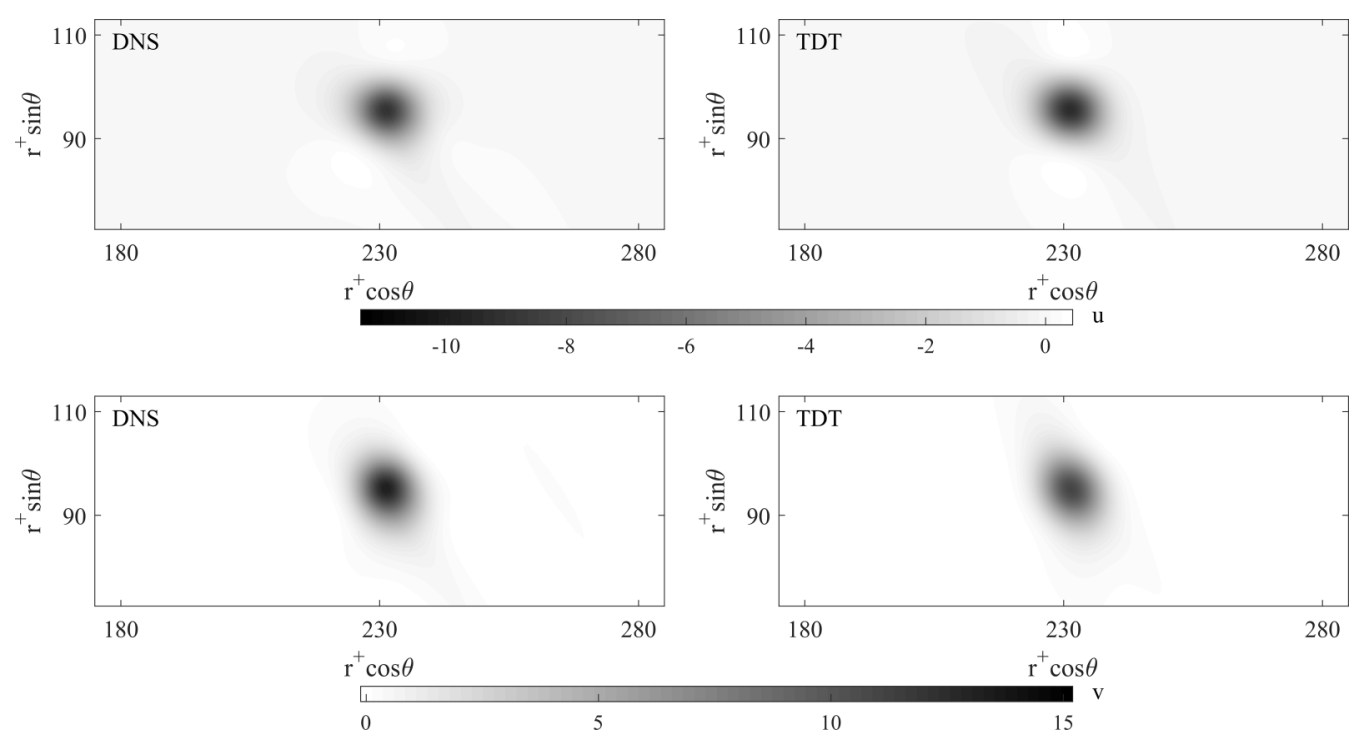

FIG. 9. Radial (top) and azimuthal (bottom) components of the perturbation for the horizontal plane at $z^{+}=0.2$ for the isolated 3D bump with $\sigma_{r}=4$ from the DNS and the TDT.

expected value of $\mathbf{u}_{1}$ can be derived in the form

$$
E\left[\mathbf{u}_{1}(\mathbf{x} ; z)\right]=E\left[\int_{-\infty}^{+\infty} K_{e}\left(\mathbf{x}-\mathbf{x}^{\prime} ; z\right) h\left(\mathbf{x}^{\prime}\right) d \mathbf{x}^{\prime}\right]=\int_{-\infty}^{+\infty} K_{e}\left(\mathbf{x}-\mathbf{x}^{\prime} ; z\right) E\left[h\left(\mathbf{x}^{\prime}\right)\right] d \mathbf{x}^{\prime} .
$$

Under the hypothesis that $E[h(\mathbf{x})]$ is independent from the position $\mathbf{x}$ and is equal to $\mu_{h}$, one obtains

$$
E\left[\mathbf{u}_{1}(\mathbf{x} ; z)\right]=\mu_{h} \int_{-\infty}^{+\infty} K_{e}\left(\mathbf{x}-\mathbf{x}^{\prime} ; z\right) d \mathbf{x}^{\prime}
$$

i.e., the mean value of velocity perturbation is proportional to the mean value of the assigned height distribution.

Similarly, the variance of the perturbed field can be related to the variance of $h(\mathbf{x})$ by considering that the variation of height for two different points along the disk surface are uncorrelated and homogeneous. For the sake of brevity, the proof is reported in Appendix B. Thus, according to the TDT presented herein, the statistics of the velocity perturbation are proportional to the height distribution ones. This relation is particularly useful because it implies that the solution obtained for $\mu_{h}$ and $\sigma_{h}$ can be rescaled for different statistics of $h(\mathbf{x})$ by separately rescaling $\mu_{\mathbf{u}}$ and $\sigma_{\mathbf{u}}$.

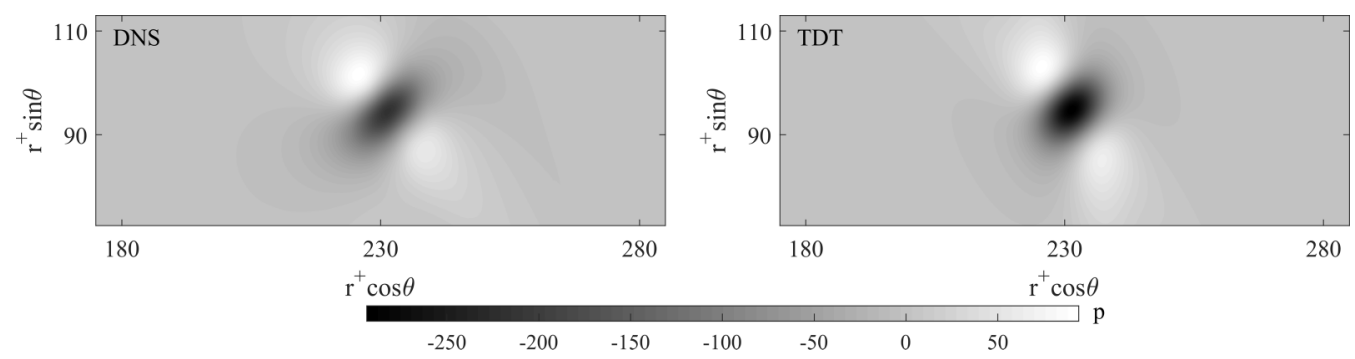

FIG. 10. Pressure correction for the horizontal plane at $z^{+}=0.2$ for the isolated 3D bump with $\sigma_{r}=4$ from the DNS and the TDT. 

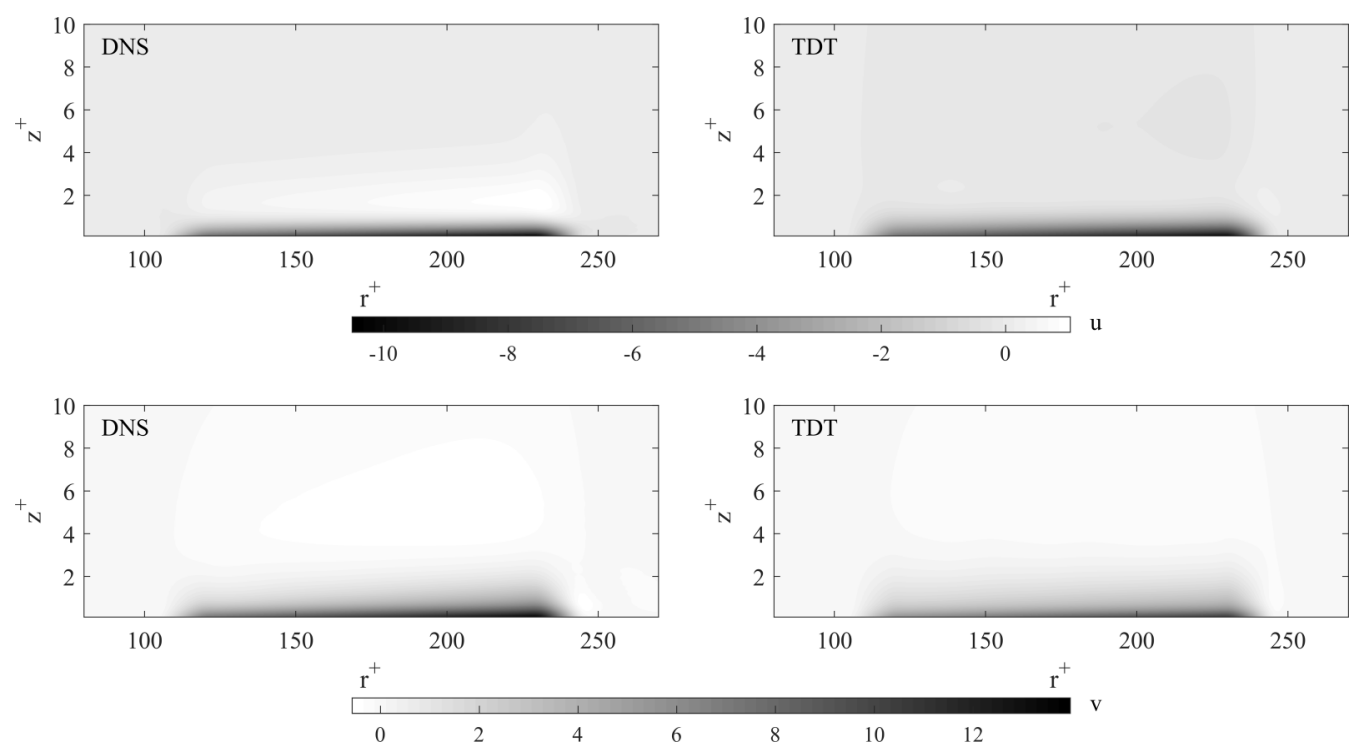

FIG. 11. Radial (top) and azimuthal (bottom) components of the perturbation for the vertical plane along the radial groove from the DNS and the TDT.

\section{B. DNSs}

A proof of concept about the use of a stochastic TDT can be provided by means of DNSs. While a Monte Carlo algorithm can be easily employed for the triple-deck model due to its fast computation, a similar approach would be prohibitively time consuming for DNSs, even for an axisymmetric roughness. Therefore, it was employed a generalized polynomial chaos (gPC) approach in order to evaluate the statistics of the perturbed field with a limited number of DNSs.
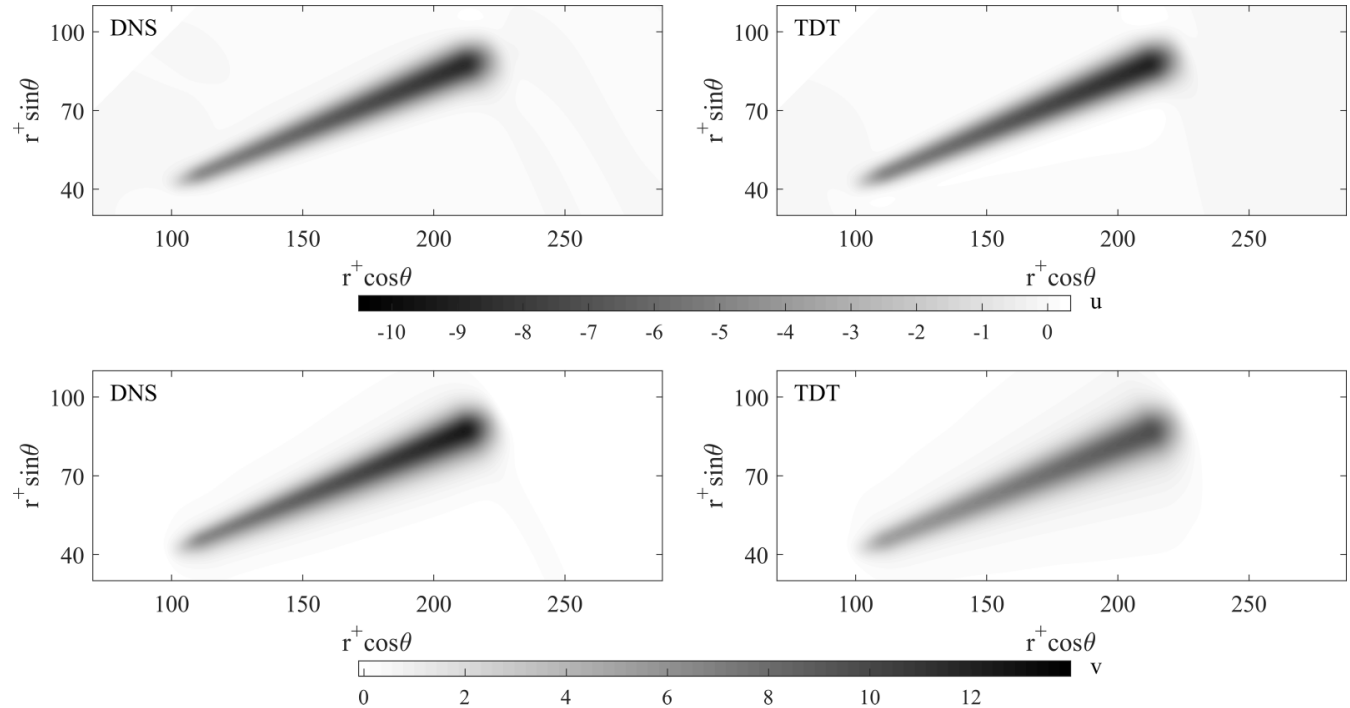

FIG. 12. Radial (top) and azimuthal (bottom) components of the perturbation for the horizontal plane at $z^{+}=0.2$ for the radial groove from the DNS and the TDT. 
The basics of gPC [21] that will be used in the following are briefly recalled here. For every point $\mathbf{x}$ of the computational domain, the gPC approach is used to evaluate a continuous response surface in the parameter space. If one defines $Q$ as a quantity of interest and $\zeta$ as the vector of independent random variables, $Q$ can be expressed as a truncated Galerkin expansion of the form

$$
Q(\zeta ; \mathbf{x})=\sum_{j=0}^{T} \alpha_{j}(\mathbf{x}) \Psi_{j}(\zeta)
$$

where $\alpha_{j}(\mathbf{x})$ and $\Psi_{j}(\zeta)$ are the $j$ th coefficients of expansion and basis function, respectively, and $T$ is the truncation order. The basis functions $\Psi_{j}(\zeta)$ are multidimensional polynomials evaluated as tensor product of one-dimensional polynomials $\psi(\zeta)$, which are suitable orthogonal polynomials chosen according to the probability density function (PDF) of each input parameter, $\zeta_{i}$ [21]. By using the orthogonality of basis functions, the coefficients $\alpha_{i}$ are computed as follows:

$$
\alpha_{j}(\mathbf{x})=\frac{\left\langle Q(\zeta ; \mathbf{x}), \Psi_{j}(\zeta)\right\rangle_{\rho}}{\left\langle\Psi_{j}(\zeta), \Psi_{j}(\zeta)\right\rangle_{\rho}} \simeq \sum_{k=1}^{N_{q}} \frac{1}{\gamma_{k}} Q\left(\zeta_{k} ; \mathbf{x}\right) \Psi_{j}\left(\zeta_{k}\right) \omega_{k},
$$

where $\langle,\rangle_{\rho}$ refers to the weighted $L_{2}$ scalar product [evaluated through the Gaussian quadrature rules associated with each one of the one-dimensional polynomials $\psi\left(\zeta_{i}\right)$ ] with weighting function $\rho$ depending of the assumed basis function. This is shown in the second part of Eq. (57), where $\zeta_{k}$ and $\omega_{k}$ are the $N_{q}$ quadrature points and weights, respectively, while $\gamma_{k}$ is the normalization factor. Thus, the model coefficients $\alpha_{j}(\mathbf{x})$ can be estimated by $N_{q}$ deterministic evaluations of $Q$ carried out on the quadrature points $\zeta_{k}$ as highlighted in Eq. (57). Once calculated the coefficients of the basis functions, the mean value $\mu_{Q}(\mathbf{x})$ and the variance $\sigma_{Q}^{2}(\mathbf{x})$ are given by

$$
\mu_{Q}(\mathbf{x})=\alpha_{0}(\mathbf{x}) \quad \sigma_{Q}^{2}(\mathbf{x})=\sum_{j=1}^{T} \alpha_{j}^{2}(\mathbf{x}) .
$$

The truncation term $T$ depends on the maximum polynomial degree $p_{i}$ for the $i$ th random parameter $\zeta_{i}$ and the number of stochastic parameters $n$.

With reference to Fig. 13, which summarizes the procedure for the stochastic validation, the roughness is represented as a sinusoidal wave in the axisymmetric domain, in the form:

$$
h\left(r^{+}\right)=\frac{h_{\max }^{+}}{2}\left[1-\cos \left(\frac{2 \pi}{\lambda} r^{+}+\phi\right)\right] .
$$

The chosen random parameters, $\zeta$, are the sinusoidal wavelength $(\lambda)$ and the phase angle, $\phi$, which works as a random shift value along radial direction does. The wavelength $\lambda$ is assumed to vary in the range $\Delta \lambda=[10,40]$ with a uniform probability density function, $p(\lambda)$. The waviness contribution is generally associated to one or two peaks at low frequencies in the Fourier spectrum of surface pattern, but it actually depends on many factors, as for instance the machining process and the chosen cutoff frequency, $\lambda_{c}$. Therefore, in order to be as general as possible, the choice of $p(\lambda)$ is conservative. The phase angle has a uniform distribution in the range $\Delta \phi=[0,2 \pi]$. This choice is justified by the fact that peaks and valleys can equally arise on every radial position.

Due to the linearity with respect to $h_{\max }^{+}$in the range of interest, the effect of an assigned $h_{\max }^{+}$can be computed a posteriori. Therefore, the maximum height $h_{\max }^{+}$is set equal to 0.1 without limiting the generality of results. This choice simplifies the problem and reduces the computational cost, because the number of deterministic simulations required rapidly increases with the dimension of $\zeta$.

Due to the fact that the length of roughness varies as an integer multiple of $\lambda$, the reference window for the results is limited to $200 \leqslant r \leqslant 300$ in order to discard waviness edges. The choice of uniform probability density functions implies the use of Legendre polynomials for both parameters in order to construct the basis functions of the expansion, $\Psi(\zeta)$ [21]. The truncation order $T$ is assessed to 24 , with a polynomial of maximum degree 4 with respect to each variable $\zeta_{i}$; thus, 

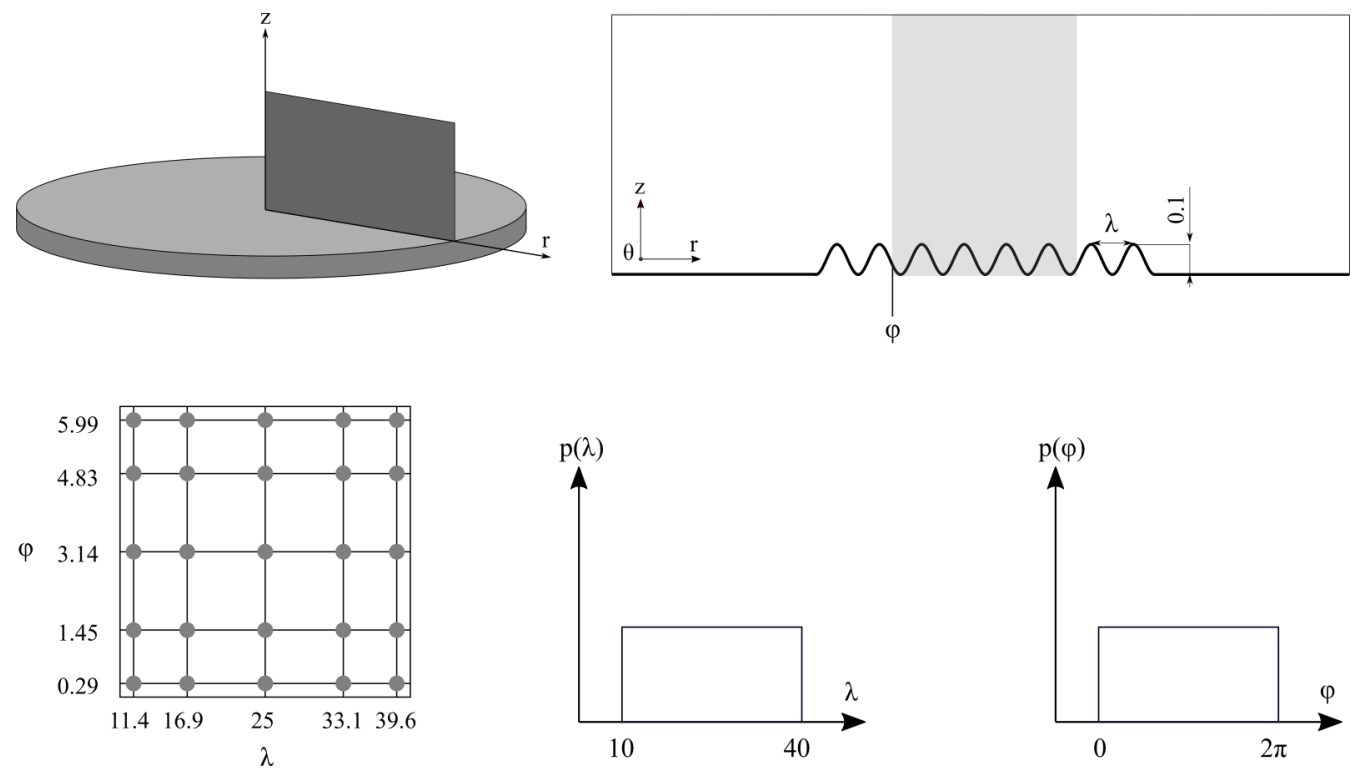

FIG. 13. Summary of numerical setup for gPC procedure. (Top) Definition of stochastic parameters. (Bottom) Tensor product grid and probability density function of parameters.

the number of required simulations $N_{q}$ is equal to 25 , as depicted with the tensor product grid in Fig. 13. The axisymmetric solver for the DNS and the setup implemented are the same as reported in Sec. IV. The resulting mean value and standard deviation of $h\left(r^{+}\right)$are independent from the radial position.
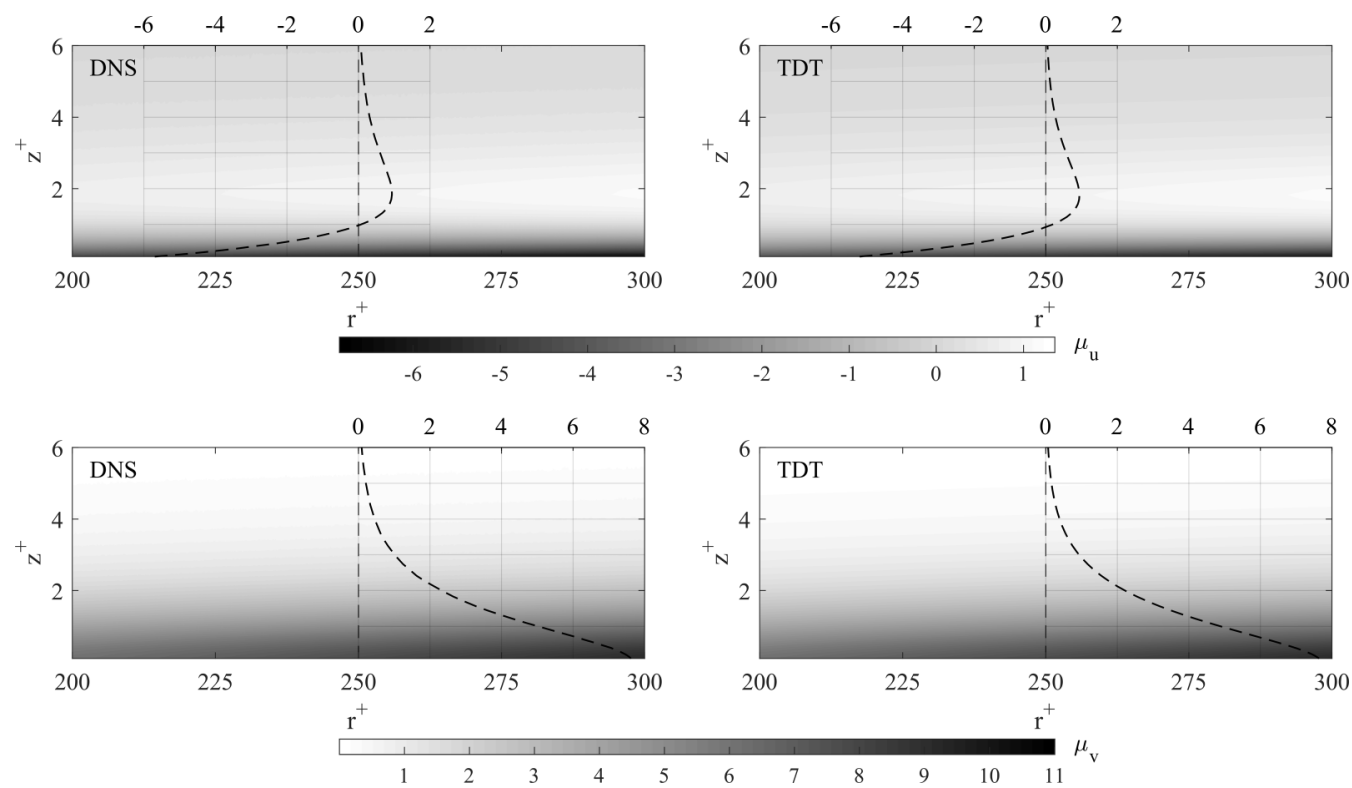

FIG. 14. Mean value of radial (top) and azimuthal (bottom) velocity components evaluated with gPC approach for sinusoidal roughness with fixed height $h_{\max }^{+}=0.1$ from the DNS and the TDT. The black dashed lines indicate the corresponding mean value of the perturbation velocity at $r^{+}=250$. 


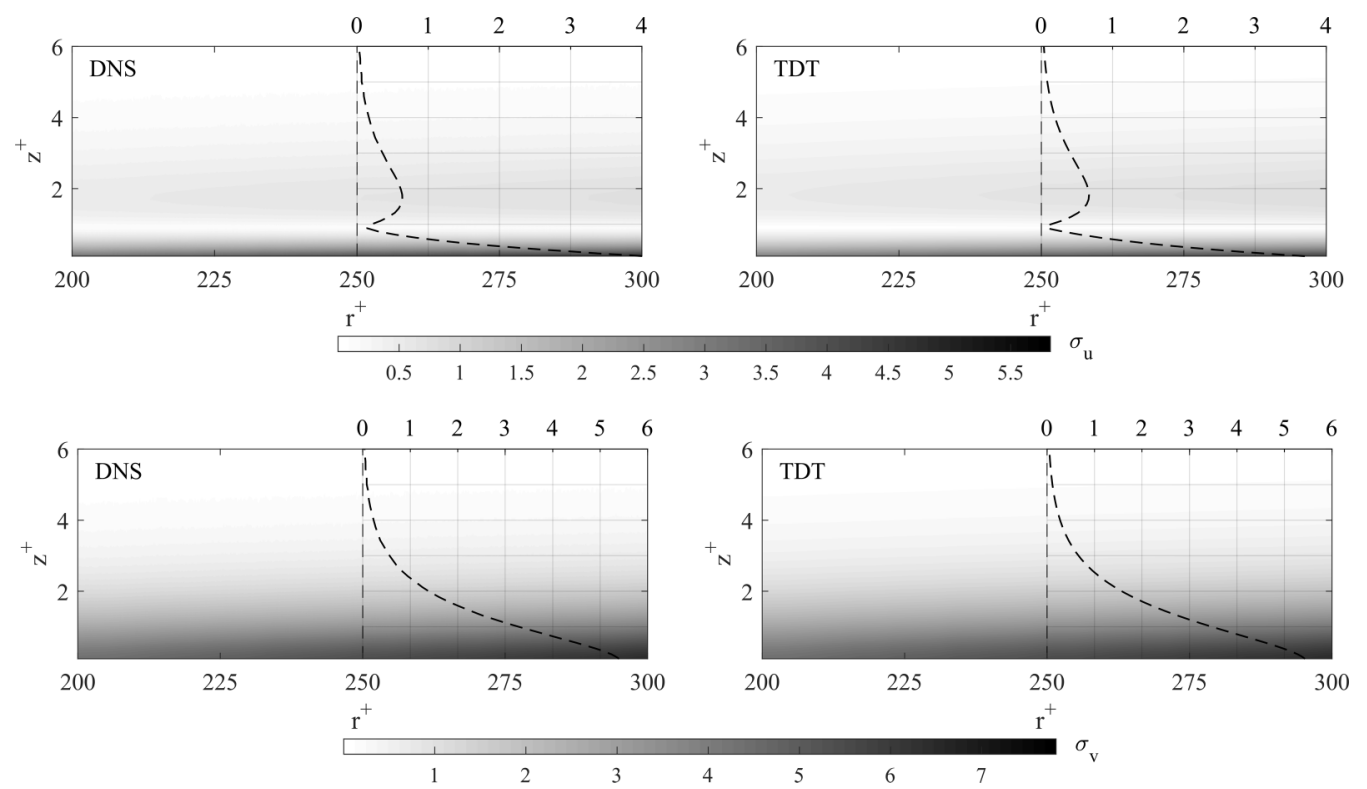

FIG. 15. Standard deviation of radial (top) and azimuthal (bottom) velocity components evaluated with gPC approach for sinusoidal roughness with fixed height $h_{\max }^{+}=0.1$ from the DNS the TDT. The black dashed lines indicate the corresponding standard deviation of perturbation velocity at $r^{+}=250$.

\section{Results}

The first comparison concerns the mean value of the overall perturbation field obtained with each model, as depicted in Fig. 14. As it is clear, there is a general good agreement between TDT and DNS perturbation field for both radial and azimuthal velocity.

The same level of accuracy is found for the standard deviation associated to the radial and azimuthal components, as depicted in Fig. 15. The main difference between TDT and DNS concerns the standard deviation of the wall-normal velocity component (not shown here): the field of $\sigma_{w}$ evaluated with the TDT model is constant along the radius, while the DNS shows a slightly increasing variability with $r^{+}$. This discrepancy is due to the fact that the TDT presented here is developed at the leading order and a more accurate representation of the wall-normal velocity requires a higher-order correction.

It is noteworthy that the mean value and the standard deviation of the perturbation field are self-similar functions that scale with the radius $r^{+}$. Thus, the resulting velocity field is a function only of $z$ coordinate. In order to provide a clearer comparison between TDT and DNS results, the black dashed lines depicted in Figs. 14 and 15 show the profile of the corresponding perturbation components for $r^{+}=250$.

Even though the case discussed may seem to be a simple representation of roughness, a similar approach can be extended to more complex patterns, because the values obtained for $\mu_{\mathbf{u}}$ and $\sigma_{\mathbf{u}}$ depend just on the statistics of the height distribution. Thus, the same results would be obtained for a more complex roughness characterized by the same mean value and standard deviation. As discussed above, the statistics of the velocity correction are proportional to the height ones. Therefore, once $\mu_{\mathbf{u}}$ and $\sigma_{\mathbf{u}}$ are evaluated for a pattern with mean value and standard deviation independent from the radial position [i.e., $\mu_{h}(\mathbf{x})=\mu_{h}$ and $\sigma_{h}(\mathbf{x})=\sigma_{h}$ ], the resulting field can be rescaled to a roughness distribution with different statistics by separately rescaling $\mu_{\mathbf{u}}$ and $\sigma_{\mathbf{u}}$. Due to its fast computation, the theory proposed here can be easily applied to roughness distribution with mean value and standard deviation, which are functions of the position $\mathbf{x}$. Indeed, it is possible to 
employ a Monte Carlo approach to evaluate the resulting mean value and standard deviation of the field.

The last feature to be discussed concerns the three-dimensional case, for which the TDT allows to evaluate also the perturbation pressure. If the statistics of the three-dimensional pattern are homogeneous along the disk surface, one finds again the same mean value and standard deviation field as for the axisymmetric domain, while the pressure gradient effect, which can be evaluated in a three-dimensional case, is null on average. Thus, in the case of homogeneous roughness with an assigned mean value and standard deviation independent from the position $\mathbf{x}$, it is possible to evaluate just the field for the axisymmetric case.

\section{CONCLUSIONS}

In this work a theory is proposed allowing us to take into account the surface roughness in the prediction of the steady laminar flow induced by a rotating disk in still fluid. The proposed theory relies on a triple-deck decomposition and leads to an analytic result, and for this reason is definitely cheap from a computational viewpoint. The theory applies to roughness elements that are much smaller than the boundary-layer height and characterized by a much longer length scale in the wall plane.

The proposed theory is validated here against the results obtained by dedicated numerical simulations in order to assess its accuracy. As reference test cases, two classes of roughness types have been considered, i.e., axisymmetric and nonaxisymmetric configurations. As concerns the axisymmetric configurations, both localized bumps and radially sinusoidal grooves have been considered. In the nonaxisymmetric case, isolated 3D bumps and localized radial grooves have been considered. Comparison between the predictions by the theory and the reference numerical simulations has been carried out on the basis of the perturbation velocity field induced by the considered roughness elements. A stochastic validation is also carried out in which the effect of a random (statistically defined) 2D roughness on the flow is quantified from a statistical view point. The analysis shows that the proposed theory generally provides results that are in good agreement with the reference simulations. In the case of elongated roughness elements, the prediction of the flow in the elongated direction is recovered in the TDT by a proposed local scaling of the predicted quantities. Finally, the TDT at the considered order predicts the effect of the pressure gradient for $3 \mathrm{D}$ roughness elements, thus providing a realistic estimation of the velocity perturbation induced upstream by the roughness elements.

The proposed theory is aimed at estimating the flow resulting from a rough rotating disk at low computational cost or even analytically, so that it can be employed in an analysis involving a wide range of roughness parameters. This is also the case for instance when the effects of a random realistic roughness is investigated, which implies a considerable number of simulations for the probabilistic characterization of the effects.

From the TDT point of view, the mean value and the standard deviation of the velocity perturbation field are proportional to the statistics of the height distribution. Hence, it is possible to exploit the linearity of the proposed model with respect to $h(\mathbf{x})$ for easily rescaling the mean value and the standard deviation of the perturbation for any homogenous height distribution. The hypotheses made do not limit the applicability of the model but rather apply to many practical cases.

\section{ACKNOWLEDGMENTS}

The authors acknowledge the CINECA award under the ISCRA initiative, for the availability of high-performance computing resources and support. The work was partly financed through the ASTRID project (2013-05786, A STudy of Rotation In Developing boundary-layer flows) funded by the Swedish Research Council VR. 

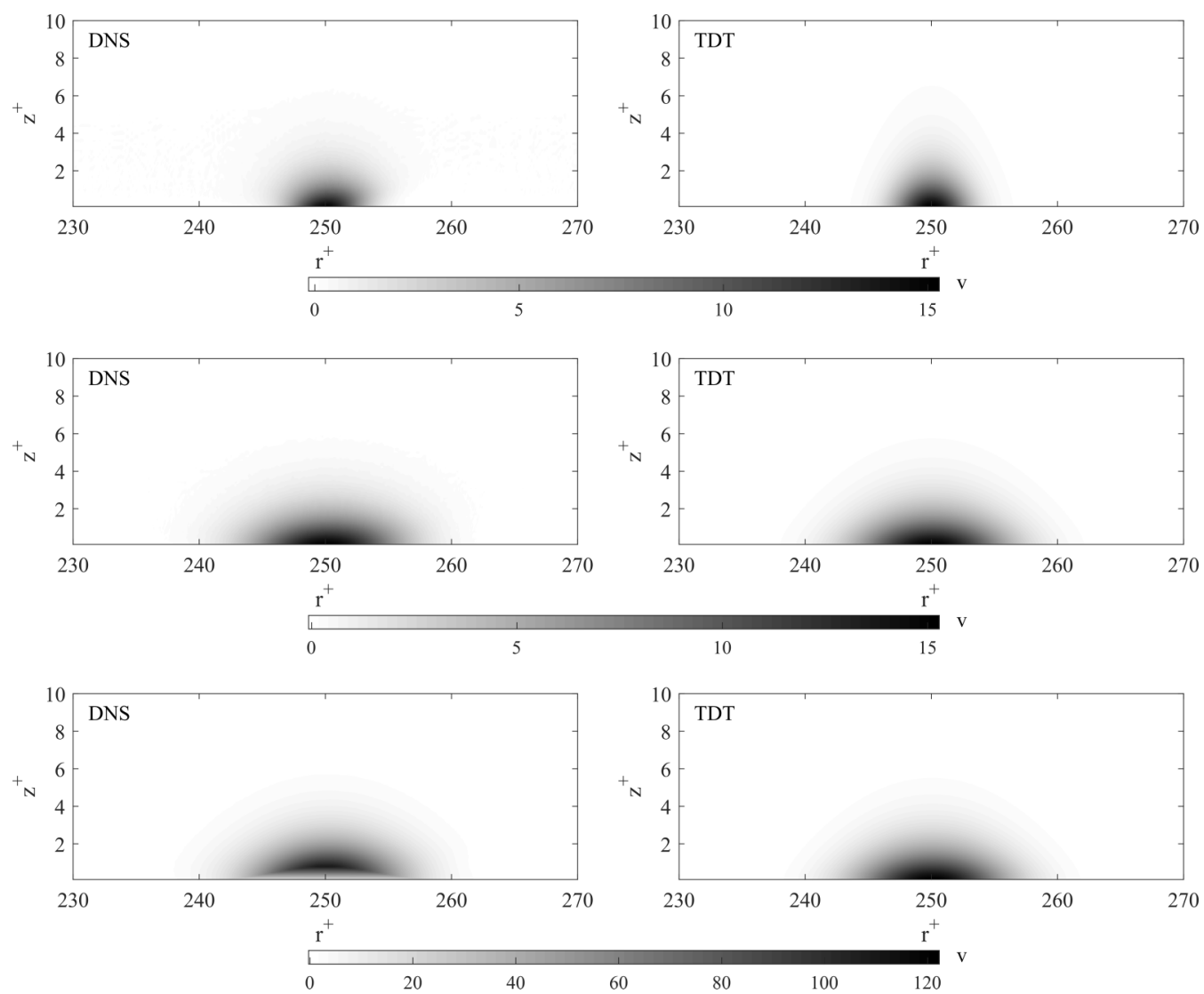

FIG. 16. Azimuthal velocity variation due to an axisymmetric bump with $h_{\max }^{+}=0.1$ and $\sigma_{r}=2$ (top) or $\sigma_{r}=4$ (middle) from the DNS and the TDT. (Bottom) azimuthal field for $h_{\max }^{+}=0.8$ and $\sigma_{r}=4$.

\section{APPENDIX A: AXISYMMETRIC BUMP}

In order to assess the validity of the proposed model with respect to the maximum height of the roughness $h_{\text {max }}$, a preliminary test in the axisymmetric domain was carried out. The tested geometry is an axisymmetric bump defined by a Gaussian function of the form

$$
h^{+}\left(r^{+}\right)=h_{\max }^{+} \exp \left[-\frac{\left(r^{+}-r_{c}^{+}\right)^{2}}{2 \sigma_{r}^{2}}\right],
$$

where $h_{\max }^{+}$is the maximum height, $r_{c}^{+}$is the Gaussian center position in the radial direction and $\sigma_{r}$ characterizes the bump width. For all the axisymmetric bump simulations, $r_{c}^{+}$was fixed to 250, while several different widths in the range $0.5 \leqslant \sigma_{r} \leqslant 4$ and heights in the range $0.01 \leqslant h_{\max }^{+} \leqslant 0.8$ were tested. The numerical setup is the same as the axisymmetric groove, as discussed in Sec. IV. For the sake of brevity, only the results of the DNSs and the TDT for three different combination of height $h_{\max }$ and width $\sigma_{r}$ are reported. Figure 16 shows the comparison between the azimuthal component of velocity variation obtained numerically and with the proposed model for two different values of width $\sigma_{r}$. The results of the TDT model are in good agreement with the numerical solution. Near the bump, the solution is well described, while small discrepancies can be found when moving along the wall-normal direction. An increase in the bump width, $\sigma_{r}$, provides a better comparison with the numerical data, as highlighted in Fig. 16. The reason for this is due to the fact that slender bumps fulfill better the hypotheses of the theory. As a final remark, the results computed in the case 
of $h_{\max }^{+}=0.8$ are reported at the bottom of Fig. 16: despite the fact that this condition is beyond the validity limits of the proposed model, the TDT is still able to provide a good approximation of the perturbation velocity with respect to DNS.

\section{APPENDIX B: STANDARD DEVIATION}

In this section, the proof of the proportionality relationship between $\sigma_{\mathbf{u}_{1}}$ and $\sigma_{h}$ is reported. As showed for the mean value in Sec. VI, we consider the relation $\hat{\mathbf{u}}_{1}(\mathbf{k} ; z)=\hat{K}_{e}(\mathbf{k} ; z) \hat{F}(\mathbf{k})$ in the Fourier space and the convolution theorem in order to rewrite the relation in the physical space. By applying the definition of variance for a random variable, one can write

$$
\sigma_{\mathbf{u}_{1}}^{2}(\mathbf{x} ; z)=E\left[\int_{-\infty}^{+\infty} K_{e}\left(\mathbf{x}-\mathbf{x}^{\prime} ; z\right)[h-E(h)]\left(\mathbf{x}^{\prime}\right) d \mathbf{x}^{\prime}\right]^{2},
$$

and by expanding the square of the convolution integral, one obtains the expression

$$
\sigma_{\mathbf{u}_{1}}^{2}(\mathbf{x} ; z)=E\left\{\int_{-\infty}^{+\infty} K_{e}\left(\mathbf{x}-\mathbf{x}^{\prime} ; z\right)[h-E(h)]\left(\mathbf{x}^{\prime}\right) d \mathbf{x}^{\prime} \int_{-\infty}^{+\infty} K_{e}\left(\mathbf{y}-\mathbf{y}^{\prime} ; z\right)[h-E(h)]\left(\mathbf{y}^{\prime}\right) d \mathbf{y}^{\prime}\right\} .
$$

One can rearrange the expression (B2) by changing the integration order under the hypotheses of Fubini's theorem obtaining

$$
\sigma_{\mathbf{u}_{1}}^{2}(\mathbf{x} ; z)=E\left\{\int_{-\infty}^{+\infty} K_{e}\left(\mathbf{x}-\mathbf{x}^{\prime} ; z\right) \int_{-\infty}^{+\infty} K_{e}\left(\mathbf{y}-\mathbf{y}^{\prime} ; z\right)[h-E(h)]\left(\mathbf{x}^{\prime}\right)[h-E(h)]\left(\mathbf{y}^{\prime}\right) d \mathbf{y}^{\prime} d \mathbf{x}^{\prime}\right\} .
$$

The integral of Eq. (B3) takes into account different variables, but one can simplify the calculus under the hypothesis that

$$
E\left\{[h-E(h)]\left(\mathbf{x}^{\prime}\right)[h-E(h)]\left(\mathbf{y}^{\prime}\right)\right\}=\sigma_{h}^{2}(\mathbf{x}) \delta\left(\mathbf{x}^{\prime}-\mathbf{y}^{\prime}\right),
$$

i.e., the statistics of $h(\mathbf{x})$ evaluated in two different points are uncorrelated. The function $\delta\left(\mathbf{x}^{\prime}-\mathbf{y}^{\prime}\right)$ is the Kronecker delta and is equal to 1 if $\mathbf{x}^{\prime}=\mathbf{y}^{\prime}$ and 0 otherwise. By substituting the expression (B4) in (B3), one can relate $\sigma_{\mathbf{u}_{1}}$ with the standard deviation of the height distribution as

$$
\sigma_{\mathbf{u}_{1}}^{2}(\mathbf{x} ; z)=E\left\{\int_{-\infty}^{+\infty} K_{e}\left(\mathbf{x}-\mathbf{x}^{\prime} ; z\right) \int_{-\infty}^{+\infty} K_{e}\left(\mathbf{y}-\mathbf{y}^{\prime} ; z\right) \sigma_{h}^{2}(\mathbf{x}) d \mathbf{y}^{\prime} d \mathbf{x}^{\prime}\right\} .
$$

Under the hypothesis that the standard deviation of $h(\mathbf{x})$ is independent from the position $\mathbf{x}$, i.e., the roughness is homogeneous, one finally obtains a formulation that shows that the standard deviation of the velocity correction $\mathbf{u}_{1}$ is directly proportional to the standard deviation of the height distribution, $\sigma_{h}$ as

$$
\sigma_{\mathbf{u}_{1}}^{2}(\mathbf{x} ; z)=\sigma_{h}^{2} \int_{-\infty}^{+\infty} K_{e}\left(\mathbf{x}-\mathbf{x}^{\prime} ; z\right) \int_{-\infty}^{+\infty} K_{e}\left(\mathbf{y}-\mathbf{y}^{\prime} ; z\right) d \mathbf{x}^{\prime} d \mathbf{y}^{\prime} .
$$

Again, we underline the fact that the hypotheses made do not limit the validity of the model in practical cases. Indeed, the complete characterization of the statistics of $h(\mathbf{x})$ is generally unnecessary or even impossible, thus only $\mu_{h}$ and $\sigma_{h}$ are provided in accordance with the surface finish tolerance.

[1] T. von Kármán, Über laminare und turbulente Reibung, Z. Angew. Math. Mech. 1, 233 (1921).

[2] N. Gregory, J. T. Stuart, and W. S. Walker, On the stability of three dimensional boundary layers with application to the flow due to a rotating disk, Philos. Trans. R. Soc. London 248, 155 (1955).

[3] M. R. Malik, The neutral curve for stationary disturbances in rotating-disk flow, J. Fluid Mech. 164, 275 (1986). 
[4] T. C. Corke and K. F. Knasiak, Stationary traveling crossflow mode interactions on a rotating disk, J. Fluid Mech. 355, 285 (1998).

[5] E. Appelquist, S. Imayama, P. H. Alfredsson, P. Schlatter, and R. J. Lingwood, Linear disturbances in the rotating-disk flow: A comparison between results from simulations, experiments and theory, Eur. J. Mech. B/Fluids 55, 170 (2016).

[6] R. J. Lingwood, Absolute instability of the boundary layer on a rotating disk, J. Fluid Mech. 299, 17 (1995).

[7] T. Corke, E. H. Matlis, and H. Othman, Transition to turbulence in rotating-disk boundary layersconvective and absolute instabilities, J. Eng. Math. 57, 253 (2007).

[8] M. Miklavčič and C. Y. Wang, The flow due to a rough rotating disk, Z. Angew. Math. Phys. 55, 235 (2004).

[9] M. S. Yoon, J. M. Hyun, and J. S. Park, Flow and heat transfer over a rotating disk with surface roughness, Int. J. Heat Fluid Flow 28, 262 (2007).

[10] S. J. Garrett, A. J. Cooper, J. H. Harris, M. Özkan, A. Segalini, and P. J. Thomas, On the stability of von Kármán rotating-disk boundary layers with radial anisotropic surface roughness, Phys. Fluids 28, 014104 (2016).

[11] A. J. Cooper, J. H. Harris, S. J. Garrett, M. Özkan, and P. J. Thomas, The effect of anisotropic and isotropic roughness on the convective stability of the rotating disk boundary layer, Phys. Fluids 27, 014107 (2015).

[12] B. Alveroglu, A. Segalini, and S. J. Garrett, The effect of surface roughness on the convective instability of the bek family of boundary-layer flows, Eur. J. Mech. B/Fluids 56, 178 (2016).

[13] A. Segalini and S. J. Garrett, On the non-parallel instability of the rotating-sphere boundary layer, J. Fluid Mech. 818, 288 (2017).

[14] A. Segalini and S. Camarri, Flow induced by a rotating cone: Base flow and convective stability analysis, Phys. Rev. Fluids 4, 084801 (2019).

[15] F. T. Smith, R. I. Sykes, and P. W. M. Brighton, A two-dimensional boundary layer encountering a threedimensional hump, J. Fluid Mech. 83, 163 (1977).

[16] A. H. Nayfeh, Triple-deck structure, Computers Fluids 20, 269 (1991).

[17] A. Segalini, J. D. Rüedi, and P. A. Monkewitz, Systematic errors of skin-friction measurements by oil-film interferometry, J. Fluid Mech. 773, 298 (2015).

[18] M. Abramowitz and I. A. Stegun, Handbook of Mathematical Functions With Formulas, Graphs, and Mathematical Tables, Applied Mathematics Series - 55 (National Bureau of Standards, Washington DC, 1972).

[19] www.freefem.org.

[20] https://nek5000.mcs.anl.gov.

[21] D. Xiu and G. Em Karniadakis, Modeling uncertainty in steady state diffusion problems via generalized polynomial chaos, Computer Methods Applied Mechanics Engineering 191, 4927 (2002). 